\title{
Biaryl synthesis via palladium-catalyzed aryne multi-component coupling
}

\author{
Jaclyn L. Henderson, ${ }^{\dagger}$ Andrew S. Edwards ${ }^{\star}$ and Michael F. Greaney ${ }^{* \dagger}$ \\ University of Edinburgh, School of Chemistry, Joseph Black Building, King's Buildings, West Mains Road, \\ Edinburgh, EH9 3JJ, UK \\ Organon Laboratories Ltd., Newhouse, Lanarkshire, ML1 5SH, UK \\ Michael.Greaney@ed.ac.uk
}

Supporting Information

$\begin{array}{ll}\text { Part A: General Experimental } & \text { S1 } \\ \text { Part B: Optimisation Data } & \text { S2 } \\ \text { Part C: Experimental Procedures } & \text { S4 } \\ \text { Part D: Spectra } & \text { S8 } \\ \text { Part E: X-ray structure of compound 20 } & \text { S32 }\end{array}$

\section{Part A: General Experimental}

NMR spectra were recorded on a Brüker dpx360 $(360 \mathrm{MHz})$ or Bruker DPX400 (400 MHz) instrument and calibrated to residual solvent peaks: proton $\left(\mathrm{CDCl}_{3} 7.26 \mathrm{ppm}\right.$ or DMSO $\left.2.50 \mathrm{ppm}\right)$ and carbon $\left(\mathrm{CDCl}_{3} 77 \mathrm{ppm}\right.$ or DMSO $39.43 \mathrm{ppm}$ ). The ${ }^{1} \mathrm{H}$ data is presented as follows: chemical shift (in ppm on the $\delta$ scale), multiplicity ( $\mathrm{s}=$ singlet, $\mathrm{d}=$ doublet, $\mathrm{t}=$ triplet, $\mathrm{q}=$ quartet, $\mathrm{m}=$ multiplet), the coupling constant $\left(\mathrm{J}\right.$, in hertz) and integration. The ${ }^{13} \mathrm{C}$ data is reported as the ppm on the $\delta$ scale followed by the interpretation. IR spectra were recorded on a JASCO FT/IR-460 plus instrument using $4 \mathrm{~mm}$ sodium chloride disks. The wavelengths of the maximum absorbance $\left(v_{\max }\right)$ are quoted in $\mathrm{cm}^{-1}$. Electrospray high resolution mass spectrometry was performed by the University of Edinburgh, using a Thermo MAT 900 double focusing mass spectrometer. The data is recorded as the ionisation method followed by the calculated and measured masses. Elemental analysis was performed using a Carlo Erba CHNS analyser at the University of St Andrews. TLC was performed on Merck 60F254 silica plates and visualised by UV light and/or anisaldehyde or potassium permanganate stains. The compounds were purified by wet flash chromatography using Merck Kieselgel 60 (particle size 35-70) silica under a positive pressure or using a Biotage or Biotage SP4 with pre-packed 12M columns. The eluent is quoted as a percentage. Solvents were dried and purified by passage through activated alumina columns using a solvent purification system from www.glasscontoursolventsystems.com or purchased (anhydrous) from Aldrich unless otherwise stated. 4-methyl-2(trimethylsilyl)phenyl trifluoromethanesulfonate (2a), trifluoro-methanesulfonic acid 6-trimethylsilanyl-1,3benzodioxol-5-yl ester and trifluoro-methanesulfonic acid 3,6-dimethyl-2-trimethylsilanyl-phenyl ester were prepared according to published procedures. ${ }^{1,2,3}$ All other chemicals were purchased from a chemical supplier and used as received.

\footnotetext{
University of Edinburgh.

Organon Laboratories Ltd.

${ }^{1}$ Peña, D.; Cobas, A.; Pérez, D; Guitián, E.; Synthesis 2002, 1454 - 1458

2 Sato, Y.; Tamura, T.; Mori, M.; Angew. Chem., Int. Ed. 2004, 43, 2436-2440.

3 Yoshida, H.; Terayama, T.; Ohshita, J.; Kunai, A.; Chem. Comm. 2004, 1980-1981.
} 


\section{Part B: Optimisation Data}

All yields refer to inseparable mixtures of $\mathbf{9}$ and $\mathbf{1 0}$ isolated by chromatography, with the 9:10 ratio being determined by ${ }^{1} \mathrm{H}$ NMR.

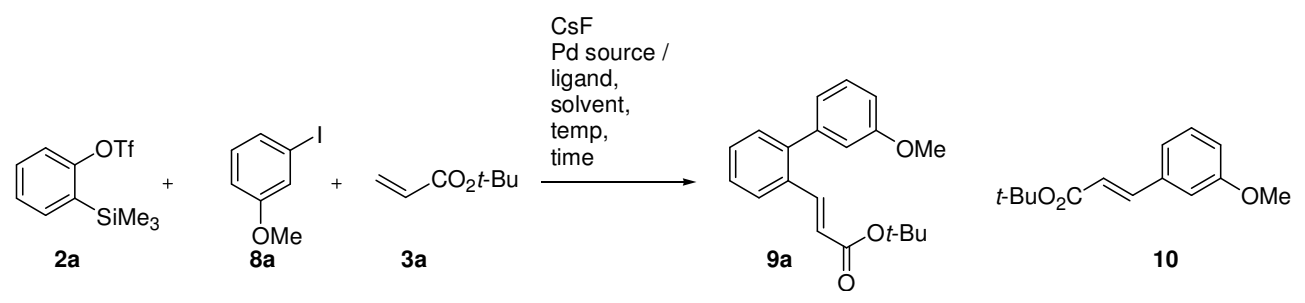

Table 1. Catalyst screen I

Table 2. Time

\begin{tabular}{ccccc}
\hline Entry & Pd Source & Ligand & $\begin{array}{c}3 \mathrm{CC}(\mathbf{9 a}) \\
(\%)\end{array}$ & $\begin{array}{c}2 \mathrm{CC}(\mathbf{1 0}) \\
(\%)\end{array}$ \\
\hline 1 & $\mathrm{Pd}\left(\mathrm{PPh}_{3}\right)_{4}$ & & 6 & 60 \\
2 & $\mathrm{Pd}(\mathrm{dppf}) \mathrm{Cl}_{2}$ & & 10 & 45 \\
3 & $\mathrm{Pd}(\mathrm{OAc})_{2}$ & dppe & 6 & 74 \\
4 & $\mathrm{Pd}(\mathrm{OAc})_{2}$ & $\mathrm{P}(o \text {-tol })_{3}$ & 23 & 24 \\
5 & $\mathrm{Pd}(\mathrm{OAc})_{2}$ & $\mathrm{P}(\text { fur })_{3}$ & 21 & 36 \\
6 & $\mathrm{Pd}(\mathrm{OAc})_{2}$ & dppp & 0 & 78 \\
7 & $\mathrm{Pd}(\mathrm{OAc})_{2}$ & $\mathrm{DPEPhos}$ & 17 & 48 \\
9 & $\mathrm{Pd}_{2}(\mathrm{dba})_{3}$ & dppe & 7 & 47 \\
10 & $\mathrm{Pd}_{2}(\mathrm{dba})_{3}$ & $\mathrm{P}(\mathrm{fur})_{3}$ & 11 & 22 \\
11 & ${\mathrm{Pd}(\mathrm{OAc})_{2}}$ & $\mathrm{P}(t \mathrm{Bu})_{3} \cdot \mathrm{HBF}_{4}$ & 18 & 35
\end{tabular}

\begin{tabular}{cccc}
\hline Entry & Time $(\mathrm{h})$ & $\begin{array}{c}3 \mathrm{CC}(\mathbf{9}) \\
(\%)\end{array}$ & $\begin{array}{c}2 \mathrm{CC} \mathrm{(10)} \\
(\%)\end{array}$ \\
\hline 1 & 1 & 23 & 10 \\
2 & 2 & 34 & 15 \\
3 & 4 & 27 & 31 \\
4 & 48 & 23 & 24
\end{tabular}

$\mathrm{Pd}(\mathrm{OAc})_{2} / \mathrm{P}(o-\text { tol })_{3}(5 \mathrm{~mol} \%), \mathrm{MeCN}, 50{ }^{\circ} \mathrm{C}$, 2:3:8 = 1:1:1

$\mathrm{MeCN}, 50{ }^{\circ} \mathrm{C}, 48 \mathrm{~h}, \mathbf{2 : 3 : 8}=1: 1: 1$

Table 3. Stoichiometry

\begin{tabular}{ccccc}
\hline Entry & Pd system & $\begin{array}{c}\text { Ligand:Pd } \\
\text { ratio }\end{array}$ & $\begin{array}{c}3 \mathrm{CC}(\mathbf{9 a}) \\
(\%)\end{array}$ & $\begin{array}{c}2 \mathrm{CC} \\
(\mathbf{1 0}) \\
(\%)\end{array}$ \\
\hline 1 & $\mathrm{Pd}(\mathrm{OAc})_{2} / \mathrm{P}(o-\text { tol })_{3}$ & $1: 1$ & 42 & 19 \\
2 & $\mathrm{Pd}(\mathrm{OAc})_{2} / \mathrm{P}(o-\text { tol })_{3}$ & $2: 1$ & 54 & 32 \\
4 & $\mathrm{Pd}(\mathrm{OAc})_{2} / \mathrm{P}(o-\text { tol })_{3}$ & $4: 1$ & 51 & 47 \\
5 & $\mathrm{Pd}(\mathrm{OAc})_{2} /$ & $1: 1$ & 50 & 20 \\
6 & $\mathrm{P}(\mathrm{tBu})_{3} \cdot \mathrm{HBF}_{4}$ & & & \\
6 & $\mathrm{Pd}(\mathrm{OAc})_{2} /$ & $2: 1$ & 48 & 27 \\
7 & $\mathrm{P}(t \mathrm{Bu})_{3} \cdot \mathrm{HBF}_{4}$ & & & \\
& $\mathrm{Pd}(\mathrm{OAc})_{2} /$ & $4: 1$ & 46 & 29
\end{tabular}

Table 4. Palladium/Ligand ratio

\begin{tabular}{cccc}
\hline \multirow{2}{*}{ Entry } & $\begin{array}{c}\text { Ratio } \\
(\mathbf{2 : 3 : 8})\end{array}$ & $\begin{array}{c}\text { 3CC (9) } \\
(\%)\end{array}$ & $\begin{array}{c}\text { 2CC (10) } \\
(\%)\end{array}$ \\
\hline 1 & $1: 1: 1$ & 34 & 15 \\
2 & $2: 1: 1$ & 35 & 19 \\
3 & $2: 1.5: 1$ & 43 & 22 \\
4 & $2: 1: 1.5$ & 42 & 14
\end{tabular}

$\mathrm{Pd}(\mathrm{OAc})_{2} / \mathrm{P}(o \text {-tol })_{3}(5 \mathrm{~mol} \%), \mathrm{MeCN}, 50^{\circ} \mathrm{C}, 2 \mathrm{~h}$

Table 5. Temperature

\begin{tabular}{cccc}
\hline Entry & Temp & $\begin{array}{c}3 C \mathrm{C}(\mathbf{9 a}) \\
(\%)\end{array}$ & $\begin{array}{c}2 \mathrm{CC}(\mathbf{1 0}) \\
(\%)\end{array}$ \\
\hline 1 & 40 & 37 & 15 \\
2 & 45 & 62 & 30 \\
4 & 50 & 55 & 36 \\
5 & $\mu \mathrm{W} 100$ & 0 &
\end{tabular}

$\mathrm{Pd}(\mathrm{OAc})_{2}, / \mathrm{P}(o-\text { tol })_{3}(5 \mathrm{~mol} \%), \mathrm{MeCN}, 4 \mathrm{~h}$ 
Table 6. Catalyst screen II

\begin{tabular}{|c|c|c|c|c|}
\hline Entry & Pd Source & Ligand & $\begin{array}{c}3 \mathrm{CC}(\mathbf{9 a}) \\
(\%)\end{array}$ & $\begin{array}{c}2 \mathrm{CC}(\mathbf{1 0}) \\
(\%)\end{array}$ \\
\hline 1 & $\mathrm{Pd}\left(\mathrm{PPh}_{3}\right)_{4}$ & & No reaction & \\
\hline 2 & $\mathrm{Pd}(\mathrm{dppf}) \mathrm{Cl}_{2}$ & & $18 \%$ & $18 \%$ \\
\hline 3 & $\mathrm{Pd}(\mathrm{OAc})_{2}$ & dppe & No reaction & \\
\hline 4 & $\mathrm{Pd}(\mathrm{OAc})_{2}$ & $\mathrm{P}(o-\text { tol })_{3}$ & $53 \%$ & $23 \%$ \\
\hline 5 & PEPPSI $^{4}$ & & No reaction & \\
\hline 6 & $\mathrm{Pd}(\mathrm{OAc})_{2}$ & TDMPP $^{5}$ & $45 \%$ & $23 \%$ \\
\hline 7 & $\mathrm{Pd}(\mathrm{OAc})_{2}$ & DPEPhos $^{6}$ & $42 \%$ & $28 \%$ \\
\hline 8 & $\mathrm{Pd}(\mathrm{OAc})_{2}$ & $\operatorname{PBiPh}(t \mathrm{Bu})_{2}{ }^{7}$ & $31 \%$ & $18 \%$ \\
\hline 9 & $\mathrm{Pd}_{2}(\mathrm{dba})_{3}$ & $\mathrm{P}(o-\text { tol })_{3}$ & $43 \%$ & $18 \%$ \\
\hline 10 & $\mathrm{Pd}_{2}(\mathrm{dba})_{3}$ & $\mathrm{P}(t \mathrm{Bu})_{3} \cdot \mathrm{HBF}_{4}$ & $16 \%$ & $28 \%$ \\
\hline 11 & $\mathrm{Pd}(\mathrm{OAc})_{2}$ & $\mathrm{P}(t \mathrm{Bu})_{3} \cdot \mathrm{HBF}_{4}$ & $48 \%$ & $29 \%$ \\
\hline 12 & Pdsuccinimide $^{8}$ & & No reaction & \\
\hline 13 & Pdsuccinimide & $\mathrm{P}(o-\text { tol })_{3}$ & No reaction & \\
\hline 14 & $\mathrm{PdCl}_{2}$ & $\mathrm{P}(o-\text { tol })_{3}$ & No reaction & \\
\hline 15 & $\mathrm{Pd}(\mathrm{PhCN})_{2} \mathrm{Cl}_{2}$ & $\mathrm{P}(o-\text { tol })_{3}$ & No reaction & \\
\hline 16 & $\mathrm{Ni}(\mathrm{dppe}) \mathrm{Cl}_{2}$ & & No reaction & \\
\hline $17^{\mathrm{a}}$ & $\begin{array}{l}\text { Hermann Beller } \\
\text { palladacycle }^{9}\end{array}$ & & $43 \%$ & $26 \%$ \\
\hline 18 & $\mathrm{Pd}(\mathrm{OAc})_{2}$ & & No reaction & \\
\hline 19 & $\mathrm{Pd}(\mathrm{OAc})_{2}$ & $\mathrm{PPh}_{3}$ & $9 \%$ & $18 \%$ \\
\hline
\end{tabular}

$\mathrm{MeCN}, 4 \mathrm{~h}, 45^{\circ} \mathrm{C}, \mathbf{2 : 3 : 8}=2: 1: 1.5$

Table 7. Solvents

\begin{tabular}{ccccc}
\hline Entry & Solvent & Ratio & $\begin{array}{c}\text { 3CC (9) } \\
(\%)\end{array}$ & $\begin{array}{c}\text { 2CC (10) } \\
(\%)\end{array}$ \\
\hline 1 & MeCN & & 62 & 30 \\
2 & DME & & 21 & 25 \\
3 & DCM & & No \\
reaction & \\
& & & 32 & 42 \\
4 & Acetone & & No & \\
5 & THF & & reaction & \\
& & & 27 & 55 \\
6 & 2-butanone & & Only 2CC & \\
7 & DCM/MeCN & $10: 1$ & 39 & 39 \\
8 & DME/MeCN & $4: 1$ & 35 & 53 \\
9 & DME/MeCN & $9: 1$ & 23 & 29 \\
10 & DME/MeCN & $99: 1$ & 23
\end{tabular}

All using $\mathrm{Pd}(\mathrm{OAc})_{2} / \mathrm{P}(o-\text { tol })_{3}$.

${ }^{4}$ O'Brien, C.J.; Kantchev, E.A.B.; Valente, C.; Hadei, N.; Chass, G.A.; Lough, A.; Hopkinson, A.C.; Organ, M.G. Chem. Eur. J. 2006, 12, 4743 - 4748.

${ }^{5}$ Wada, M.; Higashizaki, S. Chem. Commun. 1984, 7, 482-483.

${ }^{6}$ Kranenburg, M.; van der Burgt, Y.E.M.; Kamer, P.C.J.; van Leeuwen, P.W.N.M.; Goubitz, K.; Fraanje, J. Organometallics, 1995,14, 3081 - 3089.

${ }^{7}$ Aranyos, A.; Old, D.W.; Kiyomori, A.; Wolfe, J.P.; Sadighi, J.P.; Buchwald, S.L. J. Am. Chem. Soc. 1999, 121,4369 - 4378.

${ }^{8}$ Crawforth, C.M.; Burling, S,; Fairlamb, I.J.S.; Taylor, R.J.K.; Whitwood, A.C. Chem. Commun. 2003, 17, $2194-2195$.

${ }^{9}$ Herrmann, W. A.; Broßmer, C.; Öfele, K.; Reisinger, C.-P.; Priermeier, T.; Beller, M.; Fischer, H. Angew. Chem., Int. Ed. 1995, 34,1844 1847. 
Table 8. Aryl iodide performance in $2 \mathrm{CC}$ versus $3 \mathrm{CC}$ after $4 \mathrm{~h}$

\begin{tabular}{llll}
\hline entry & aryl iodide & yield in $2 \mathrm{CC}^{a}(\%)$ & yield in $3 \mathrm{CC}^{b}(\%)$ \\
\hline 1 & 22 & 62 \\
2 & $13\left(91^{c}\right)$ & 86 \\
3
\end{tabular}

[a] Reactions carried out as in Scheme 2 over $4 \mathrm{~h}$ with the omission of aryne

precursor 2. [b] Reaction conditions as Scheme 2. [c] Yield after 24 h. nd = not determined.

\section{Part C: Experimental Procedures: General method for 3-component coupling involving aryl iodides}

The aryl iodide $\left(0.31 \mathrm{mmol}, 1.5\right.$ equiv) was added to a stirred suspension of $\mathrm{Pd}(\mathrm{OAc})_{2}(2 \mathrm{mg}, 5 \mathrm{~mol} \%), \mathrm{P}(\mathrm{o} \text {-tol })_{3}$ $(6.1 \mathrm{mg}, 10 \mathrm{~mol} \%)$ and $\mathrm{CsF}$ (6 equiv) in dry $\mathrm{MeCN}(1 \mathrm{~mL})$ under $\mathrm{N}_{2}$ in a carousel tube and heated to $45^{\circ} \mathrm{C}$. This was followed by the addition of tert-butyl acrylate 3a $(0.21 \mathrm{mmol}, 1$ equiv) and 2-(trimethylsilyl)phenyl trifluoromethane sulfonate (2a) $\left(0.21 \mathrm{mmol}, 1\right.$ equiv). The reaction was stirred at $45^{\circ} \mathrm{C}$ for 1 hour after which a second portion of 2-(trimethylsilyl)phenyl trifluoromethane sulfonate (2a) $(0.21 \mathrm{mmol}, 1$ equiv) was added. The reaction was stirred for a further 3 hours. The reaction was diluted with DCM and filtered through a short plug of silica then concentrated. Purification on silica, eluting with 30\% - 50\% DCM/hexanes yielded the title compounds.

(E)-3-(3'-Methoxy-biphenyl-2-yl)-acrylic acid (acid of 9a): Isolated in a yield of 53\%, following treatment of the esters 9a and 10 with TFA/DCM 1:1 overnight at room temperature. Concentration in vacuo and recrystallization from EtOAc afforded the acid as a colourless solid. m.p. (DCM) $131{ }^{\circ} \mathrm{C} .{ }^{1} \mathrm{H}$ NMR $\left(\mathrm{CDCl}_{3}, 400 \mathrm{MHz}\right) \delta 7.68(\mathrm{~d}$, $1 \mathrm{H}, \mathrm{CH}=\mathrm{CH}, \mathrm{J}=15.8 \mathrm{~Hz}), 7.63(\mathrm{~d}, 2 \mathrm{H}, \operatorname{Ar} H, \mathrm{~J}=7.6 \mathrm{~Hz}), 7.39-7.27(\mathrm{~m}, 4 \mathrm{H}, \operatorname{Ar} H), 6.89-6.80(\mathrm{~m}, 3 \mathrm{H}, \operatorname{Ar} H)$, $6.33(\mathrm{~d}, 1 \mathrm{H}, \mathrm{CH}=\mathrm{CH}, \mathrm{J}=15.9 \mathrm{~Hz}), 3.76\left(\mathrm{~s}, 3 \mathrm{H}, \mathrm{OCH}_{3}\right) ;{ }^{13} \mathrm{C} \mathrm{NMR}\left(\mathrm{CDCl}_{3}, 100 \mathrm{MHz}\right) \delta 172.0$ (quat), 159.5 (quat), $146.1(\mathrm{CH}), 143.1$ (quat), 141.1 (quat), 132.2 (quat), $130.4(\mathrm{CH}), 130.2(\mathrm{CH}), 129.3(\mathrm{CH}), 127.8(\mathrm{CH}), 127.0(\mathrm{CH})$, $122.4(\mathrm{CH}), 118.1(\mathrm{CH}), 115.4(\mathrm{CH}), 113.5(\mathrm{CH}), 55.3\left(\mathrm{CH}_{3}\right)$; IR $\left(\mathrm{film} / \mathrm{cm}^{-1}\right)$ 2300, 1707, 1626, 1472, 1421, 1320, 1214; HRMS $\left(\mathrm{ES}^{+}\right)$cald for $\mathrm{C}_{16} \mathrm{H}_{14} \mathrm{O}_{3}:(\mathrm{M}+\mathrm{H})^{+} 254.09375$. Found: 254.09491 .

(E)-3-(4'-Bromo-biphenyl-2-yl)-acrylic acid tert-butyl ester (9b): Isolated in a yield of $63 \%$ as a colourless solid. m.p. (DCM) $52-54{ }^{\circ} \mathrm{C} ;{ }^{1} \mathrm{H}$ NMR $\left(\mathrm{CDCl}_{3}, 400 \mathrm{MHz}\right) \delta 7.70(\mathrm{~d}, 1 \mathrm{H}, \mathrm{ArH}, \mathrm{J}=7.6 \mathrm{~Hz}), 7.61-7.55(\mathrm{~m}, 3 \mathrm{H}, \mathrm{CH}=\mathrm{CH}$, $\operatorname{ArH}), 7.44-7.36(\mathrm{~m}, 2 \mathrm{H}, \operatorname{ArH}), 7.32(\mathrm{~m}, 1 \mathrm{H}, \mathrm{ArH}), 7.19(\mathrm{~d}, 2 \mathrm{H}, \mathrm{ArH}, \mathrm{J}=8.4 \mathrm{~Hz}), 6.34(\mathrm{~d}, 1 \mathrm{H}, \mathrm{CH}=\mathrm{CH}, \mathrm{J}=16.0$ $\mathrm{Hz}$ ), 1.49 (s, 9H, $\left.\mathrm{CCH}_{3}\right) ;{ }^{13} \mathrm{C} \mathrm{NMR}\left(\mathrm{CDCl}_{3}, 100.55 \mathrm{MHz}\right) \delta 166.1$ (quat), $142.0(\mathrm{CH}), 141.6$ (quat), 138.9 (quat), 132.7 (quat), $131.4(2 \mathrm{C}, \mathrm{CH}), 131.4(2 \mathrm{C}, \mathrm{CH}), 130.3(\mathrm{CH}), 129.7(\mathrm{CH}), 127.9(\mathrm{CH}), 126.7(\mathrm{CH}), 121.9$ (quat), $121.3(\mathrm{CH}), 80.5$ (quat), $28.2\left(3 \mathrm{C}, \mathrm{CH}_{3}\right)$; IR $\left(\mathrm{film} / \mathrm{cm}^{-1}\right)$ 1707, 1632, 1474, 1321, 1150; HRMS (ES $\left.{ }^{+}\right)$cald for $\mathrm{C}_{19} \mathrm{H}_{19}{ }^{81} \mathrm{BrO}_{2}:(\mathrm{M}+\mathrm{H})^{+} 360.05425$. Found: 360.05424 .

(E)-3-(3'-Chloro-biphenyl-2-yl)-acrylic acid tert-butyl ester (9c): Isolated in a yield of $68 \%$ as a colourless oil. ${ }^{1} \mathrm{H}$ NMR $\left(\mathrm{CDCl}_{3}, 400 \mathrm{MHz}\right) \delta 7.70(\mathrm{~m}, 1 \mathrm{H}, \mathrm{ArH}), 7.59(\mathrm{~d}, 1 \mathrm{H}, \mathrm{CH}=\mathrm{CH}, \mathrm{J}=15.9 \mathrm{~Hz}), 7.42-7.31(\mathrm{~m}, 6 \mathrm{H}, \mathrm{ArH})$, $7.19(\mathrm{~m}, 1 \mathrm{H}, \mathrm{ArH}), 6.33(\mathrm{~d}, 1 \mathrm{H}, \mathrm{CH}=\mathrm{CH}, \mathrm{J}=15.9 \mathrm{~Hz}), 1.49\left(\mathrm{~s}, 9 \mathrm{H}, \mathrm{CCH}_{3}\right) ;{ }^{13} \mathrm{C} \mathrm{NMR}\left(\mathrm{CDCl}_{3}, 100 \mathrm{MHz}\right) \delta 166.0$ (quat), $141.8(\mathrm{CH}), 141.3$ (quat), 134.2 (quat), 132.7 (quat), $130.3(\mathrm{CH}), 129.7(\mathrm{CH}), 129.6(\mathrm{CH}), 129.4(\mathrm{CH})$, $128.1(\mathrm{CH}), 127.6(\mathrm{CH}), 126.7(\mathrm{CH}), 121.5(\mathrm{CH}), 80.4$ (quat), $28.1\left(3 \mathrm{C}, \mathrm{CH}_{3}\right)$; IR (film/ $\left./ \mathrm{cm}^{-1}\right) 2977,1709,1633$, 1466, 1367, 1321, 1151; HRMS $\left(\mathrm{ES}^{+}\right)$cald for $\mathrm{C}_{15} \mathrm{H}_{10}{ }^{35} \mathrm{ClO}_{2}$ : $\left(\mathrm{M}-{ }^{+} \mathrm{Bu}\right)^{+}$257.03638. Found: 257.03584.

(E)-3-(3'-Fluoro-biphenyl-2-yl)-acrylic acid tert-butyl ester (9d): Isolated in a yield of $74 \%$ as a colourless oil. ${ }^{1} \mathrm{H}$ NMR $\left(\mathrm{CDCl}_{3}, 400 \mathrm{MHz}\right) \delta 7.70(\mathrm{dd}, 1 \mathrm{H}, \mathrm{ArH}, \mathrm{J}=1.6,7.2 \mathrm{~Hz}), 7.60(\mathrm{~d}, 1 \mathrm{H}, \mathrm{CH}=\mathrm{CH}, \mathrm{J}=15.6 \mathrm{~Hz}), 7.44-7.32$ $(\mathrm{m}, 4 \mathrm{H}, \operatorname{ArH}), 7.09$ (dd, 2H, $\mathrm{ArH}, \mathrm{J}=2.4,8.0 \mathrm{~Hz}), 7.04(\mathrm{~m}, 1 \mathrm{H}, \mathrm{ArH}), 6.33(\mathrm{~d}, 1 \mathrm{H}, \mathrm{CH}=\mathrm{CH}, \mathrm{J}=16.0 \mathrm{~Hz}) 1.48(\mathrm{~s}$, $\left.9 \mathrm{H}, \mathrm{CCH}_{3}\right) ;{ }^{13} \mathrm{C} \mathrm{NMR}\left(\mathrm{CDCl}_{3}, 100.55 \mathrm{MHz}\right) \delta 166.0$ (quat), $162.6(\mathrm{CF}, \mathrm{d}, \mathrm{J}=246.5 \mathrm{~Hz}$ ), 142.2 (quat, $\mathrm{d}, \mathrm{J}=7.7 \mathrm{~Hz}$ ), $141.9(\mathrm{CH}), 141.5$ (quat), 132.7 (quat), $130.3(\mathrm{CH}), 129.7(\mathrm{CH}, \mathrm{d}, \mathrm{J}=8.5 \mathrm{~Hz}), 129.6(\mathrm{CH}) 128.0(\mathrm{CH}), 126.7(\mathrm{CH})$, $125.6(\mathrm{CH}, \mathrm{d}, \mathrm{J}=2.9 \mathrm{~Hz}), 121.4(\mathrm{CH}), 116.7(\mathrm{CH}, \mathrm{d}, \mathrm{J}=21.7 \mathrm{~Hz}), 114.4(\mathrm{CH}, \mathrm{d}, \mathrm{J}=21.0 \mathrm{~Hz}), 80.4($ quat $), 28.1$ $\left(3 \mathrm{C}, \mathrm{CH}_{3}\right) ;{ }^{19} \mathrm{~F}$ NMR $\left(\mathrm{CDCl}_{3}, 377 \mathrm{MHz}\right) \delta-113.2$; IR $\left(\mathrm{film} / \mathrm{cm}^{-1}\right) 2978,1708,1633,1473,1367,1322,1151$; HRMS (ES ${ }^{+}$) cald for $\mathrm{C}_{19} \mathrm{H}_{19} \mathrm{FO}_{2}:(\mathrm{M}+\mathrm{H})^{+} 298.13636$. Found: 298.13562.

(E)-3-(2'-Nitro-biphenyl-2-yl)-acrylic acid tert-butyl ester (9e): Isolated in a yield of 56\% as a slightly yellow oil. ${ }^{1} \mathrm{H}$ NMR $\left(\mathrm{CDCl}_{3}, 400 \mathrm{MHz}\right) \delta 8.04(\mathrm{dd}, 1 \mathrm{H}, \operatorname{ArH}, \mathrm{J}=0.8,8.0 \mathrm{~Hz}), 7.72(\mathrm{~m}, 1 \mathrm{H}, \operatorname{Ar} H), 7.65(\mathrm{dt}, 1 \mathrm{H}, \operatorname{Ar} H, \mathrm{~J}=1.0$, $7.2 \mathrm{~Hz}), 7.55$ (dt, $1 \mathrm{H}, \mathrm{ArH}, \mathrm{J}=1.2,7.8 \mathrm{~Hz}), 7.43-7.37(\mathrm{~m}, 2 \mathrm{H}, \mathrm{CH}=\mathrm{CH}, \mathrm{Ar} H), 7.34-7.30(\mathrm{~m}, 2 \mathrm{H}, \operatorname{Ar} H), 7.19-$ $7.17(\mathrm{~m}, 1 \mathrm{H}, \mathrm{Ar} H), 6.30(\mathrm{~d}, 1 \mathrm{H}, \mathrm{CH}=\mathrm{CH}, \mathrm{J}=15.6 \mathrm{~Hz}) 1.45\left(\mathrm{~s}, 9 \mathrm{H}, \mathrm{CCH}_{3}\right) ;{ }^{13} \mathrm{C} \mathrm{NMR}\left(\mathrm{CDCl}_{3}, 100.55 \mathrm{MHz}\right) \delta$ 
165.8 (quat), 149.2 (quat), $140.5(\mathrm{CH}), 138.4$ (quat), 134.9 (quat), 132.9 (quat), $132.7(\mathrm{CH}), 132.6(\mathrm{CH}), 129.6$ $(\mathrm{CH}), 129.1(\mathrm{CH}), 128.9(\mathrm{CH}), 128.5(\mathrm{CH}), 126.3(\mathrm{CH}), 124.5(\mathrm{CH}), 121.9(\mathrm{CH}), 80.5$ (quat) $28.1\left(3 \mathrm{C}, \mathrm{CH}_{3}\right)$; IR $\left(\right.$ film $\left./ \mathrm{cm}^{-1}\right)$ 2978, 1706, 1634, 1574, 1351, 1323, 1150; HRMS $\left(\mathrm{ES}^{+}\right)$cald for $\mathrm{C}_{19} \mathrm{H}_{19} \mathrm{NO}_{4}:(\mathrm{M}+\mathrm{H})^{+} 325.13086$. Found: 325.12939 .

(E)-3-(3'-Nitro-biphenyl-2-yl)-acrylic acid tert-butyl ester (9f): Isolated in a yield of $85 \%$ as a colourless solid. m.p. (DCM) $143{ }^{\circ} \mathrm{C} ;{ }^{1} \mathrm{H}$ NMR $\left(\mathrm{CDCl}_{3}, 400 \mathrm{MHz}\right) \delta 8.27-8.21(\mathrm{~m}, 2 \mathrm{H}, \mathrm{ArH}), 7.72(\mathrm{~m}, 1 \mathrm{H}, \mathrm{ArH}), 7.66-7.60(\mathrm{~m}$, $2 \mathrm{H}, \operatorname{Ar} H), 7.50(\mathrm{~d}, 1 \mathrm{H}, \mathrm{CH}=\mathrm{CH}, \mathrm{J}=16.0 \mathrm{~Hz}), 7.47-7.44(\mathrm{~m}, 2 \mathrm{H}, \mathrm{ArH}), 7.36(\mathrm{~m}, 1 \mathrm{H}, \mathrm{ArH}), 6.36(\mathrm{~d}, 1 \mathrm{H}, \mathrm{CH}=\mathrm{CH}$, $\mathrm{J}=16.0 \mathrm{~Hz}$ ), $1.47\left(\mathrm{~s}, 9 \mathrm{H}, \mathrm{CCH}_{3}\right) ;{ }^{13} \mathrm{C} \mathrm{NMR}\left(\mathrm{CDCl}_{3}, 100.55 \mathrm{MHz}\right) \delta 165.8$ (quat), 148.3 (quat), 141.7 (quat), 141.1 $(\mathrm{CH}), 140.0$ (quat), $135.8(\mathrm{CH}), 132.9$ (quat), $130.3(\mathrm{CH}), 129.9(\mathrm{CH}), 129.2(\mathrm{CH}), 128.7(\mathrm{CH}), 127.0(\mathrm{CH}), 124.5$ $(\mathrm{CH}), 122.4(\mathrm{CH}), 122.3(\mathrm{CH}), 80.6$ (quat), $28.1\left(3 \mathrm{C}, \mathrm{CH}_{3}\right)$; IR (film/ $\left.\mathrm{cm}^{-1}\right)$ 1707, 1633, 1530, 1351, 1322; HRMS $\left(\mathrm{ES}^{+}\right)$cald for $\mathrm{C}_{19} \mathrm{H}_{19} \mathrm{NO}_{4}:(\mathrm{M}+\mathrm{H})^{+}$325.13086. Found: 325.13082.

(E)-3-(3'-Cyano-biphenyl-2-yl)-acrylic acid tert-butyl ester (9g): Isolated in a yield of $90 \%$ as a colourless solid. m.p. (DCM) $93{ }^{\circ} \mathrm{C} ;{ }^{1} \mathrm{H}$ NMR $\left(\mathrm{CDCl}_{3}, 400 \mathrm{MHz}\right) \delta 7.73-7.70(\mathrm{~m}, 2 \mathrm{H}, \mathrm{ArH}), 7.62$ (bs, $\left.1 \mathrm{H}, \mathrm{ArH}\right), 7.56-7.55$ (m, $2 \mathrm{H}, \operatorname{Ar} H), 7.48(\mathrm{~d}, 1 \mathrm{H}, \mathrm{CH}=\mathrm{CH}, \mathrm{J}=16.0 \mathrm{~Hz}), 7.44-7.41(\mathrm{~m}, 2 \mathrm{H}, \mathrm{ArH}), 7.31(\mathrm{~m}, 1 \mathrm{H}, \operatorname{ArH}) 6.35(\mathrm{~d}, 1 \mathrm{H}, \mathrm{CH}=\mathrm{CH}, \mathrm{J}$ $=15.6 \mathrm{~Hz}), 1.48\left(\mathrm{~s}, 9 \mathrm{H}, \mathrm{CCH}_{3}\right) ;{ }^{13} \mathrm{C} \mathrm{NMR}\left(\mathrm{CDCl}_{3}, 90.55 \mathrm{MHz}\right) \delta 165.8$ (quat), 141.3 (quat), $141.2(\mathrm{CH}), 140.2$ (quat), $134.2(\mathrm{CH}), 133.1(\mathrm{CH}), 132.7$ (quat), $131.1(\mathrm{CH}), 130.2(2 \mathrm{C}, \mathrm{CH}), 129.9(\mathrm{CH}), 128.6(\mathrm{CH}), 126.9(\mathrm{CH})$, $122.1(\mathrm{CH}), 118.6$ (quat), 112.6 (quat), 80.7 (quat), 28.1 (3C, $\left.\mathrm{CH}_{3}\right)$; IR (film/ $\mathrm{cm}^{-1}$ ) 2978, 1706, 1633, 1472, 1367, 1322, 1150; HRMS (ES ${ }^{+}$cald for $\mathrm{C}_{20} \mathrm{H}_{19} \mathrm{NO}_{2}:(\mathrm{M}+\mathrm{H})^{+}$305.14103. Found: 305.14073.

2'-((E)-2-tert-Butoxycarbonyl-vinyl)-biphenyl-3-carboxylic acid ethyl ester (9h): Isolated in a yield of 70\% as a colourless oil. ${ }^{1} \mathrm{H}$ NMR $\left(\mathrm{CDCl}_{3}, 400 \mathrm{MHz}\right) \delta 8.07(\mathrm{~m}, 1 \mathrm{H}, \mathrm{ArH}), 8.02(\mathrm{~s}, 1 \mathrm{H}, \mathrm{ArH}), 7.71(\mathrm{~d}, 1 \mathrm{H}, \operatorname{Ar} H, \mathrm{~J}=7.2 \mathrm{~Hz})$, $7.56(\mathrm{~d}, 1 \mathrm{H}, \mathrm{CH}=\mathrm{CH}, \mathrm{J}=16.0 \mathrm{~Hz}), 7.51-7.50(\mathrm{~m}, 2 \mathrm{H}, \operatorname{ArH}), 7.44-7.36(\mathrm{~m}, 3 \mathrm{H}, \operatorname{ArH}) 6.33(\mathrm{~d}, 1 \mathrm{H}, \mathrm{CH}=\mathrm{CH}, \mathrm{J}=$ $16.0 \mathrm{~Hz}), 4.39\left(\mathrm{q}, 2 \mathrm{H}, \mathrm{CH}_{2} \mathrm{CH}_{3}\right), 1.46\left(\mathrm{~s}, 9 \mathrm{H}, \mathrm{CCH}_{3}\right), 1.39\left(\mathrm{t}, 3 \mathrm{H}, \mathrm{CH}_{2} \mathrm{CH}_{3}\right) ;{ }^{13} \mathrm{C} \mathrm{NMR}\left(\mathrm{CDCl}_{3}, 100.55 \mathrm{MHz}\right) \delta$ 166.4 (quat), 166.0 (quat), $141.9(\mathrm{CH}), 141.8$ (quat), 140.3 (quat), $134.2(\mathrm{CH}), 132.8$ (quat), $130.7(\mathrm{CH}), 130.6$ $(\mathrm{CH}), 130.4(\mathrm{CH}), 129.7(\mathrm{CH}), 128.7(\mathrm{CH}), 128.2(\mathrm{CH}), 128.0(\mathrm{CH}), 126.7(\mathrm{CH}), 121.5(\mathrm{CH}), 80.4$ (quat), 61.0 $\left(\mathrm{CH}_{2}\right), 28.1\left(3 \mathrm{C}, \mathrm{CH}_{3}\right), 14.3\left(\mathrm{CH}_{3}\right)$; IR (film/ $\left.\mathrm{cm}^{-1}\right)$ 2979, 1706, 1633, 1472, 1367, 1239, 1150; HRMS (ES ${ }^{+}$) cald for $\mathrm{C}_{18} \mathrm{H}_{16} \mathrm{O}_{4}:\left(\mathrm{M}-{ }^{\mathrm{t}} \mathrm{Bu}\right)^{+}$296.10431. Found: 296.10316.

(E)-3-(4'-Trifluoromethyl-biphenyl-2-yl)-acrylic acid tert-butyl ester (9i): Isolated in a yield of $86 \%$ as a colourless oily solid. m.p. (DCM) $59{ }^{\circ} \mathrm{C} ;{ }^{1} \mathrm{H}$ NMR $\left(\mathrm{CDCl}_{3}, 400 \mathrm{MHz}\right) \delta 7.74-7.69(\mathrm{~m}, 3 \mathrm{H}, \mathrm{ArH}), 7.56(\mathrm{~d}, 1 \mathrm{H}$, $\mathrm{CH}=\mathrm{CH}, \mathrm{J}=16.0 \mathrm{~Hz}), 7.45-7.41(\mathrm{~m}, 4 \mathrm{H}, \mathrm{Ar} H), 7.34(\mathrm{dd}, 1 \mathrm{H}, \mathrm{Ar} H, \mathrm{~J}=1.8,7.0 \mathrm{~Hz}), 6.36(\mathrm{~d}, 1 \mathrm{H}, \mathrm{CH}=\mathrm{CH}, \mathrm{J}=$ $16.0 \mathrm{~Hz}) 1.48\left(\mathrm{~s}, 9 \mathrm{H}, \mathrm{CCH}_{3}\right) ;{ }^{13} \mathrm{C} \mathrm{NMR}\left(\mathrm{CDCl}_{3}, 100.55 \mathrm{MHz}\right) \delta 166.0$ (quat), 143.7 (quat), $141.6(\mathrm{CH}), 141.3$ (quat), 132.7 (quat), $130.3(\mathrm{CH}), 130.1(2 \mathrm{C}, \mathrm{CH}), 129.7(\mathrm{CH}), 129.7$ (quat, d, J = 32.5 Hz) $128.3(\mathrm{CH}), 126.8(\mathrm{CH})$, 125.2 (CH), $125.2(\mathrm{CH}), 124.2\left(\mathrm{CF}_{3}, \mathrm{q}, \mathrm{J}=271.9 \mathrm{~Hz}\right), 121.7(\mathrm{CH}), 80.6$ (quat) $28.1\left(3 \mathrm{C}, \mathrm{CH}_{3}\right) ;{ }^{19} \mathrm{~F} \mathrm{NMR}\left(\mathrm{CDCl}_{3}\right.$, $377 \mathrm{MHz}) \delta$-62.5; IR (film/ $\left.\mathrm{cm}^{-1}\right)$ 2979, 1708, 1634, 1321, 1152, 1127; HRMS (ES ${ }^{+}$cald for $\mathrm{C}_{20} \mathrm{H}_{19} \mathrm{~F}_{3} \mathrm{O}_{2}:(\mathrm{M}+\mathrm{H})^{+}$ 348.13317. Found: 348.13285 .

(E)-3-(3',4'-Dichloro-biphenyl-2-yl)-acrylic acid tert-butyl ester (9j): Isolated in a yield of $88 \%$ as a colourless solid. m.p. (DCM) $55^{\circ} \mathrm{C} .{ }^{1} \mathrm{H} \mathrm{NMR}\left(\mathrm{CDCl}_{3}, 400 \mathrm{MHz}\right) \delta 7.69(\mathrm{~m}, 1 \mathrm{H}, \mathrm{ArH}), 7.55(\mathrm{~d}, 1 \mathrm{H}, \mathrm{CH}=\mathrm{CH}, \mathrm{J}=15.9 \mathrm{~Hz})$, $7.50(\mathrm{~d}, 1 \mathrm{H}, \operatorname{Ar} H, \mathrm{~J}=8.2 \mathrm{~Hz}), 7.44-7.38(\mathrm{~m}, 3 \mathrm{H}, \operatorname{Ar} H), 7.30(\mathrm{~m}, 1 \mathrm{H}, \operatorname{Ar} H), 7.14(\mathrm{dd}, 1 \mathrm{H}, \operatorname{Ar} H, \mathrm{~J}=2.1,8.2 \mathrm{~Hz})$, $6.34(\mathrm{~d}, 1 \mathrm{H}, \mathrm{CH}=\mathrm{CH}, \mathrm{J}=15.9 \mathrm{~Hz}), 1.49\left(\mathrm{~s}, 9 \mathrm{H}, \mathrm{CCH}_{3}\right) ;{ }^{13} \mathrm{C} \mathrm{NMR}\left(\mathrm{CDCl}_{3}, 100.55 \mathrm{MHz}\right) \delta 166.0$ (quat), 141.5 $(\mathrm{CH}), 140.1$ (quat), 140.0 (quat), 132.7 (quat), 132.5 (quat), 131.9 (quat), $131.4(\mathrm{CH}), 130.2(2 \mathrm{C}, \mathrm{CH}), 129.8(\mathrm{CH})$, $129.2(\mathrm{CH}), 128.4(\mathrm{CH}), 126.8(\mathrm{CH}), 121.8(\mathrm{CH}), 80.7$ (quat), $28.2\left(3 \mathrm{C}, \mathrm{CH}_{3}\right)$; IR (film/ $\left.\mathrm{cm}^{-1}\right)$ 3399, 2976, 1707, 1634, 1463, 1321, 1151; HRMS $\left(\mathrm{ES}^{+}\right)$cald for $\mathrm{C}_{19} \mathrm{H}_{19}{ }^{35} \mathrm{Cl}_{2} \mathrm{O}_{2}$ : $(\mathrm{M}+\mathrm{H})^{+}$348.06784. Found: 348.06741. This compound was then treated with TFA and coupled to thiophene-2-sulfonamide in the literature preparation of compound 11 .

(E)-3-(4'-Chloro-biphenyl-2-yl)-acrylic acid tert-butyl ester (9k): Isolated in a yield of $74 \%$ as a colourless solid. m.p. (DCM) $99{ }^{\circ} \mathrm{C} .{ }^{1} \mathrm{H}$ NMR $\left(\mathrm{CDCl}_{3}, 400 \mathrm{MHz}\right) \delta 7.70(\mathrm{~d}, 1 \mathrm{H}, \mathrm{ArH}, \mathrm{J}=7.4 \mathrm{~Hz}), 7.60(\mathrm{~d}, 1 \mathrm{H}, \mathrm{CH}=\mathrm{CH}, \mathrm{J}=15.9$ $\mathrm{Hz}), 7.42-7.38(\mathrm{~m}, 4 \mathrm{H}, \operatorname{Ar} H), 7.32(\mathrm{~m}, 1 \mathrm{H}, \operatorname{Ar} H), 7.26-7.24(\mathrm{~m}, 2 \mathrm{H}, \operatorname{Ar} H), 6.35(\mathrm{~d}, 1 \mathrm{H}, \mathrm{CH}=\mathrm{CH}, \mathrm{J}=16.0 \mathrm{~Hz})$, 1.49 (s, 9H, $\left.\mathrm{CCH}_{3}\right) ;{ }^{13} \mathrm{C} \mathrm{NMR}\left(\mathrm{CDCl}_{3}, 100.55 \mathrm{MHz}\right) \delta 166.1$ (quat), $142.0(\mathrm{CH}), 141.5$ (quat), 138.4 (quat), 133.7 (quat), 132.7 (quat), $131.0(2 \mathrm{C}, \mathrm{CH}), 130.3(\mathrm{CH}), 129.7(\mathrm{CH}), 128.5(2 \mathrm{C}, \mathrm{CH}), 127.9(\mathrm{CH}), 126.7(\mathrm{CH}), 121.3$ (CH), 80.5 (quat), $28.2\left(3 \mathrm{C}, \mathrm{CH}_{3}\right)$; IR (film/ $\left./ \mathrm{cm}^{-1}\right)$ 2977, 1708, 1632, 1474, 1367, 1322, 1152; HRMS (ES ${ }^{+}$) cald for $\mathrm{C}_{19} \mathrm{H}_{19}{ }^{35} \mathrm{ClO}_{2}$ : $(\mathrm{M}+\mathrm{H})^{+}$314.10681. Found: 314.10598. 
(E)-tert-butyl 3-(2-(thiophen-2-yl)phenyl)acrylate (9I): Isolated in a yield of 50\% as a slightly yellow oil. ${ }^{1} \mathrm{H}$ $\operatorname{NMR}\left(\mathrm{CDCl}_{3}, 400 \mathrm{MHz}\right) \delta 7.91(\mathrm{~d}, 1 \mathrm{H}, \mathrm{CH}=\mathrm{CH}, \mathrm{J}=16.0 \mathrm{~Hz}), 7.66(\mathrm{dd}, 1 \mathrm{H}, \mathrm{ArH}, \mathrm{J}=1.4,7.4 \mathrm{~Hz}), 7.49(\mathrm{dd}, 1 \mathrm{H}$, $\operatorname{Ar} H, \mathrm{~J}=1.4,7.4 \mathrm{~Hz}), 7.41-7.33(\mathrm{~m}, 3 \mathrm{H}, \operatorname{Ar} H), 7.12(\mathrm{dd}, 1 \mathrm{H}, \operatorname{Ar} H, \mathrm{~J}=3.6,5.2 \mathrm{~Hz}), 7.04(\mathrm{dd}, 1 \mathrm{H}, \operatorname{Ar} H, \mathrm{~J}=0.8$, $3.6 \mathrm{~Hz}) 6.35(\mathrm{~d}, 1 \mathrm{H}, \mathrm{CH}=\mathrm{CH}, \mathrm{J}=16.0 \mathrm{~Hz}), 1.52\left(\mathrm{~s}, 9 \mathrm{H}, \mathrm{CCH}_{3}\right) ;{ }^{13} \mathrm{C} \mathrm{NMR}\left(\mathrm{CDCl}_{3}, 100.55 \mathrm{MHz}\right) \delta 166.2$ (quat), $142.4(\mathrm{CH}), 141.3$ (quat), 135.0 (quat), 133.4 (quat), $130.9(\mathrm{CH}), 129.5(\mathrm{CH}), 128.2(\mathrm{CH}), 128.0(\mathrm{CH}), 127.6(\mathrm{CH})$, $127.1(\mathrm{CH}), 126.3(\mathrm{CH}), 121.7(\mathrm{CH}), 80.4$ (quat), $28.3\left(3 \mathrm{C}, \mathrm{CH}_{3}\right) ; \mathrm{IR}\left(\mathrm{film} / \mathrm{cm}^{-1}\right)$ 2976, 1708, 1631, 1367, 1320; HRMS (ES ${ }^{+}$) cald for $\mathrm{C}_{17} \mathrm{H}_{18} \mathrm{O}_{2} \mathrm{~S}:(\mathrm{M}+\mathrm{H})^{+} 286.10220$. Found: 286.10086.

(E)-3-(3'-Chloro-4-methyl-biphenyl-2-yl)-acrylic acid tert-butyl ester and (E)-3-(3'-Chloro-5-methylbiphenyl-2-yl)-acrylic acid tert-butyl ester (9m): Isolated as a 50:50 mixture of the title compounds in a yield of $70 \%$ as a colourless oil. ${ }^{1} \mathrm{H}$ NMR $\left(\mathrm{CDCl}_{3}, 400 \mathrm{MHz}\right) \delta 7.62-7.51(\mathrm{~m}, 2 \mathrm{H}, \mathrm{ArH}), 7.36-7.31(\mathrm{~m}, 3 \mathrm{H}, \mathrm{Ar} H), 7.26-$ $7.14(\mathrm{~m}, 3 \mathrm{H}, \mathrm{ArH}), 6.32(\mathrm{~d}, 0.5 \mathrm{H}, \mathrm{CH}=\mathrm{CH}, \mathrm{J}=15.9 \mathrm{~Hz}), 6.29(\mathrm{~d}, 0.5 \mathrm{H}, \mathrm{CH}=\mathrm{CH}, \mathrm{J}=15.8 \mathrm{~Hz}), 2.42(\mathrm{~s}, 1.5 \mathrm{H}$, $\left.\mathrm{ArCH}_{3}\right), 2.40\left(\mathrm{~s}, 1.5 \mathrm{H}, \mathrm{ArCH}_{3}\right), 1.49\left(\mathrm{~s}, 4.5 \mathrm{H}, \mathrm{CCH}_{3}\right), 1.48\left(\mathrm{~s}, 4.5 \mathrm{H}, \mathrm{CCH}_{3}\right) ;{ }^{13} \mathrm{C} \mathrm{NMR}\left(\mathrm{CDCl}_{3}, 100 \mathrm{MHz}\right) \delta 166.2$ (quat), 166.1 (quat), $142.0(\mathrm{CH}), 142.0$ (quat), 141.8 (quat), $141.8(\mathrm{CH}), 141.3$ (quat), 140.0 (quat), 138.6(quat), 137.9 (quat), 134.2 (2C, quat), 132.5 (quat), $130.9(\mathrm{CH}), 130.6(\mathrm{CH}), 130.2(\mathrm{CH}), 129.9$ (quat), $129.8(\mathrm{CH}), 129.7$ $(\mathrm{CH}), 129.4(2 \mathrm{C}, \mathrm{CH}), 129.0(\mathrm{CH}), 128.1(\mathrm{CH}), 128.1(\mathrm{CH}), 127.5(\mathrm{CH}), 127.4(\mathrm{CH}), 127.2(\mathrm{CH}), 126.6(\mathrm{CH})$, $121.2(\mathrm{CH}), 120.5(\mathrm{CH}), 80.4$ (quat), 80.3 (quat), $28.2\left(3 \mathrm{C}, \mathrm{CH}_{3}\right), 21.3\left(\mathrm{CH}_{3}\right), 21.1\left(\mathrm{CH}_{3}\right) ; \mathrm{IR}\left(\mathrm{film} / \mathrm{cm}^{-1}\right) 2977$, 1708, 1632, 1468, 1367, 1321, 1198; HRMS (ES ${ }^{+}$cald for $\mathrm{C}_{20} \mathrm{H}_{21}{ }^{35} \mathrm{ClO}_{2}$ : $(\mathrm{M}+\mathrm{H})^{+}$328.12246. Found: 328.12167.

(E)-3-(3'-Chloro-3,6-dimethyl-biphenyl-2-yl)-acrylic acid tert-butyl ester (9n): Isolated in a yield of $65 \%$ as a colourless oil. ${ }^{1} \mathrm{H}$ NMR $\left(\mathrm{CDCl}_{3}, 400 \mathrm{MHz}\right) \delta 7.32-7.25(\mathrm{~m}, 3 \mathrm{H}, \mathrm{ArH}, \mathrm{CH}=\mathrm{CH}), 7.14-7.11(\mathrm{~m}, 3 \mathrm{H}, \mathrm{ArH}), 6.99$ $(\mathrm{m}, 1 \mathrm{H}, \mathrm{ArH}), 5.67(\mathrm{~d}, 1 \mathrm{H}, \mathrm{CH}=\mathrm{CH}, \mathrm{J}=16.4 \mathrm{~Hz}), 2.39\left(\mathrm{~s}, 3 \mathrm{H}, \mathrm{ArCH}_{3}\right), 2.03\left(\mathrm{~s}, 3 \mathrm{H}, \mathrm{ArCH}_{3}\right), 1.41\left(\mathrm{~s}, 9 \mathrm{H}, \mathrm{CCH}_{3}\right)$; ${ }^{13} \mathrm{C} \mathrm{NMR}\left(\mathrm{CDCl}_{3}, 100.55 \mathrm{MHz}\right) \delta 165.8$ (quat), $142.4(\mathrm{CH}), 142.2$ (quat), 140.4 (quat), 134.2 (quat), 134.1 (quat), 133.7 (quat), 133.4 (quat), $130.1(\mathrm{CH}), 130.0(\mathrm{CH}), 129.8(\mathrm{CH}), 129.5(\mathrm{CH}), 128.0(\mathrm{CH}), 127.1(\mathrm{CH}), 125.7(\mathrm{CH})$, 80.2 (quat), $28.1\left(3 \mathrm{C}, \mathrm{CH}_{3}\right), 21.2\left(\mathrm{CH}_{3}\right), 20.5\left(\mathrm{CH}_{3}\right) ; \mathrm{IR}\left(\mathrm{film} / \mathrm{cm}^{-1}\right)$ 2976, 1710, 1633, 1367, 1307; HRMS (ES $\left.{ }^{+}\right)$ cald for $\mathrm{C}_{21} \mathrm{H}_{23}{ }^{35} \mathrm{ClO}_{2}$ : (M+H) ${ }^{+} 342.13811$. Found: 342.13729 .

(E)-3-[6-(4-Trifluoromethyl-phenyl)-benzo[1,3]dioxol-5-yl]-acrylic acid tert-butyl ester (9o): Isolated in a yield of $38 \%$ as a colourless solid. m.p. (DCM) $145-147{ }^{\circ} \mathrm{C} .{ }^{1} \mathrm{H}$ NMR $\left(\mathrm{CDCl}_{3}, 400 \mathrm{MHz}\right) \delta 7.68(\mathrm{~d}, 2 \mathrm{H}, \mathrm{ArH}, \mathrm{J}=8.1$ $\mathrm{Hz}), 7.45(\mathrm{~d}, 1 \mathrm{H}, \mathrm{CH}=\mathrm{CH}, \mathrm{J}=15.8 \mathrm{~Hz}), 7.39(\mathrm{~d}, 2 \mathrm{H}, \operatorname{ArH}, \mathrm{J}=8.0 \mathrm{~Hz}), 7.18(\mathrm{~s}, 1 \mathrm{H}, \operatorname{Ar} H), 6.78(\mathrm{~s}, 1 \mathrm{H}, \operatorname{ArH}), 6.21$ $(\mathrm{d}, 1 \mathrm{H}, \mathrm{CH}=\mathrm{CH}, \mathrm{J}=15.8 \mathrm{~Hz}), 6.05\left(\mathrm{~s}, 2 \mathrm{H}, \mathrm{CH}_{2}\right), 1.46\left(\mathrm{~s}, 9 \mathrm{H}, \mathrm{CCH}_{3}\right) ;{ }^{13} \mathrm{C} \mathrm{NMR}\left(\mathrm{CDCl}_{3}, 100.55 \mathrm{MHz}\right) \delta 166.2$ (quat), 149.1 (quat), 148.1 (quat), 143.5 (quat), $141.3(\mathrm{CH}), 136.7$ (quat), $130.2(2 \mathrm{C}, \mathrm{CH}), 129.7$ (d, quat, $\mathrm{J}=32.6$ $\mathrm{Hz}), 126.7(\mathrm{CH}), 125.2(\mathrm{q}, 2 \mathrm{C}, \mathrm{CH}, \mathrm{J}=3.7 \mathrm{~Hz}), 124.2\left(\mathrm{q}, \mathrm{CF}_{3}, \mathrm{~J}=272.1 \mathrm{~Hz}\right), 119.6(\mathrm{CH}), 110.1(\mathrm{CH}), 105.8(\mathrm{CH})$, $101.8\left(\mathrm{CH}_{2}\right), 80.4$ (quat), $28.1\left(3 \mathrm{C}, \mathrm{CH}_{3}\right)$; IR $\left(\mathrm{film} / \mathrm{cm}^{-1}\right)$ 2927, 1703, 1615, 1483, 1368, 1326, 1152; HRMS (ES $)$ cald for $\mathrm{C}_{21} \mathrm{H}_{19} \mathrm{~F}_{3} \mathrm{O}_{4}:(\mathrm{M}+\mathrm{H})^{+}$392.12300. Found: 392.12323 .

3'-Chloro-2-[(E)-2-(4-methoxy-phenyl)-vinyl]-biphenyl (9p): Isolated in a yield of 70\% as a colourless oil. ${ }^{1} \mathrm{H}$ $\operatorname{NMR}\left(\mathrm{CDCl}_{3}, 400 \mathrm{MHz}\right) \delta 7.75(\mathrm{~d}, 1 \mathrm{H}, \mathrm{ArH}, \mathrm{J}=7.6 \mathrm{~Hz}), 7.41-7.21(\mathrm{~m}, 8 \mathrm{H}, \mathrm{ArH}, \mathrm{CH}=\mathrm{CH}), 7.03(\mathrm{~d}, 2 \mathrm{H}, \mathrm{ArH}, \mathrm{J}$ $=3.6 \mathrm{~Hz}), 6.97(\mathrm{~d}, 1 \mathrm{H}, \operatorname{Ar} H, \mathrm{~J}=7.6 \mathrm{~Hz}), 6.90(\mathrm{t}, 1 \mathrm{H}, \operatorname{ArH}, \mathrm{J}=2.0 \mathrm{~Hz}), 6.79(\mathrm{dd}, 1 \mathrm{H}, \operatorname{ArH}, \mathrm{J}=1.8,8.0 \mathrm{~Hz}), 3.80(\mathrm{~s}$, $\left.3 \mathrm{H}, \mathrm{OCH}_{3}\right) ;{ }^{13} \mathrm{C} \mathrm{NMR}\left(\mathrm{CDCl}_{3}, 100.55 \mathrm{MHz}\right) \delta 159.9$ (quat), 142.7 (quat), 139.7 (quat), 138.9 (quat), 135.4 (quat), 134.1 (quat), $130.1(\mathrm{CH}), 129.9(\mathrm{CH}), 129.8(\mathrm{CH}), 129.6(\mathrm{CH}), 129.3(\mathrm{CH}), 128.2(\mathrm{CH}), 128.1(\mathrm{CH}), 127.7(\mathrm{CH})$, $127.6(\mathrm{CH}), 127.3(\mathrm{CH}), 126.0(\mathrm{CH}), 119.3(\mathrm{CH}), 113.3(\mathrm{CH}), 112.0(\mathrm{CH}), 55.2\left(\mathrm{CH}_{3}\right) ; \mathrm{IR}\left(\mathrm{film} / \mathrm{cm}^{-1}\right) 2917,2852$, 1647, 1599, 1464, 1431, 1267; HRMS $\left(\mathrm{ES}^{+}\right)$cald for $\mathrm{C}_{21} \mathrm{H}_{17}{ }^{35} \mathrm{ClO}$ : $(\mathrm{M}+\mathrm{H})^{+} 320.09624$. Found: 320.09655.

(E)-1-(3'-Chloro-biphenyl-2-yl)-hex-1-en-3-one (9q): Isolated in a yield of 38\% as a colourless oil. ${ }^{1} \mathrm{H}$ NMR $\left(\mathrm{CDCl}_{3}, 400 \mathrm{MHz}\right) \delta 7.71(\mathrm{dd}, 1 \mathrm{H}, \mathrm{ArH}, \mathrm{J}=1.6,7.2 \mathrm{~Hz}) 7.51(\mathrm{~d}, 1 \mathrm{H}, \mathrm{CH}=\mathrm{CH}, \mathrm{J}=16.0 \mathrm{~Hz}), 7.45-7.34(\mathrm{~m}, 6 \mathrm{H}$, $\operatorname{ArH}), 7.20(\mathrm{~m}, 1 \mathrm{H}, \mathrm{ArH}), 6.65(\mathrm{~d}, 1 \mathrm{H}, \mathrm{CH}=\mathrm{CH}, \mathrm{J}=16.4 \mathrm{~Hz}), 2.5\left(\mathrm{t}, \mathrm{CH}_{2}, \mathrm{~J}=7.4 \mathrm{~Hz}\right), 1.65$ (sextet, $\mathrm{CH}_{2}, \mathrm{~J}=7.4$ $\mathrm{Hz}$ ), $0.93\left(\mathrm{t}, 3 \mathrm{H}, \mathrm{CH}_{3}, \mathrm{~J}=7.4 \mathrm{~Hz}\right) ;{ }^{13} \mathrm{C} \mathrm{NMR}\left(\mathrm{CDCl}_{3}, 100.55 \mathrm{MHz}\right) \delta 200.6$ (quat), 141.8 (quat), 141.5 (quat), 141.1 $(\mathrm{CH}), 134.4$ (quat), 132.9 (quat), $130.3(\mathrm{CH}), 130.0(\mathrm{CH}), 129.8(\mathrm{CH}), 129.5(\mathrm{CH}), 128.3(\mathrm{CH}), 128.2(\mathrm{CH}), 128.1$ $(\mathrm{CH}), 127.8(\mathrm{CH}), 126.9(\mathrm{CH}), 42.2\left(\mathrm{CH}_{2}\right), 18.0\left(\mathrm{CH}_{2}\right), 13.8\left(\mathrm{CH}_{3}\right) ; \mathrm{IR}\left(\mathrm{film} / \mathrm{cm}^{-1}\right)$ 2961, 1667, 1609, 1465, 1406 , 1185; HRMS (ES ${ }^{+}$) cald for $\mathrm{C}_{18} \mathrm{H}_{17}{ }^{35} \mathrm{ClO}:(\mathrm{M}+\mathrm{H})^{+} 284.09624$. Found: 284.09611

(E)-3-(3'-Chloro-biphenyl-2-yl)- $\mathbf{N}, \mathbf{N}$-dimethyl-acrylamide (9r): Isolated in a yield of $74 \%$ as a slightly yellow oil. ${ }^{1} \mathrm{H}$ NMR $\left(\mathrm{CDCl}_{3}, 400 \mathrm{MHz}\right) \delta 7.66(\mathrm{~m}, 1 \mathrm{H}, \mathrm{ArH}), 7.59(\mathrm{~d}, 1 \mathrm{H}, \mathrm{CH}=\mathrm{CH}, \mathrm{J}=15.5 \mathrm{~Hz}), 7.42-7.37(\mathrm{~m}, 2 \mathrm{H}$, $\operatorname{ArH}), 7.35-7.30(\mathrm{~m}, 4 \mathrm{H}, \mathrm{ArH}), 7.19(\mathrm{~m}, 1 \mathrm{H}, \mathrm{ArH}), 6.74(\mathrm{~d}, 1 \mathrm{H}, \mathrm{CH}=\mathrm{CH}, \mathrm{J}=15.4 \mathrm{~Hz}), 3.10\left(\mathrm{bs}, 3 \mathrm{H}, \mathrm{NCH}_{3}\right), 3.02$ (bs, $\left.3 \mathrm{H}, \mathrm{NCH}_{3}\right) ;{ }^{13} \mathrm{C} \mathrm{NMR}\left(\mathrm{CDCl}_{3}, 100 \mathrm{MHz}\right) \delta 166.5$ (quat), 142.1 (quat), 141.0 (quat), $140.5(\mathrm{CH}), 134.2$ (quat), 133.6 (quat), $130.4(\mathrm{CH}), 129.5(\mathrm{CH}), 129.1(\mathrm{CH}), 128.1(\mathrm{CH}), 128.0(\mathrm{CH}), 127.5(\mathrm{CH}), 127.0(\mathrm{CH}), 119.6(\mathrm{CH})$, 
$37.4\left(\mathrm{CH}_{3}\right), 35.8\left(\mathrm{CH}_{3}\right)$; IR (film/ $\left.\mathrm{cm}^{-1}\right)$ 2927, 1650, 1609, 1393, 1140; HRMS (ES $)$ cald for $\mathrm{C}_{17} \mathrm{H}_{16}{ }^{35} \mathrm{ClNO}_{2}$ : $(\mathrm{M}+\mathrm{H})^{+}$285.09149. Found: 285.09024.

(E)-1-Morpholin-4-yl-3-(4'-trifluoromethyl-biphenyl-2-yl)-propenone (9s): Isolated in a yield of 91\% as a colourless solid. m.p. (DCM) $154-156{ }^{\circ} \mathrm{C} .{ }^{1} \mathrm{H}$ NMR $\left(\mathrm{CDCl}_{3}, 400 \mathrm{MHz}\right) \delta 7.70-7.67(\mathrm{~m}, 3 \mathrm{H}, \mathrm{ArH}), 7.64(\mathrm{~d}, 1 \mathrm{H}$, $\mathrm{CH}=\mathrm{CH}, \mathrm{J}=15.6 \mathrm{~Hz}), 7.46-7.42(\mathrm{~m}, 4 \mathrm{H}, \mathrm{ArH}), 7.33(\mathrm{~m}, 1 \mathrm{H}, \mathrm{ArH}), 6.70(\mathrm{~d}, 1 \mathrm{H}, \mathrm{CH}=\mathrm{CH}, \mathrm{J}=15.4 \mathrm{~Hz}), 3.69-$ 3.57 (bm, 8H, $\left.\mathrm{CH}_{2}\right) ;{ }^{13} \mathrm{C} \mathrm{NMR}\left(\mathrm{CDCl}_{3}, 100.55 \mathrm{MHz}\right) \delta 165.2$ (quat), 143.9 (quat), $141.4(\mathrm{CH}), 141.0$ (quat), 133.4 (quat), $130.5(\mathrm{CH}), 130.0(2 \mathrm{C}, \mathrm{CH}), 129.6(\mathrm{~d}$, quat, J = 32.6 Hz), $129.5(\mathrm{CH}), 128.3(\mathrm{CH}), 127.1(\mathrm{CH}), 125.3(\mathrm{q}, 2 \mathrm{C}$, $\mathrm{CH}, \mathrm{J}=7.2 \mathrm{~Hz}), 124.1\left(\mathrm{q}, \mathrm{CF}_{3}, \mathrm{~J}=272 \mathrm{~Hz}\right), 118.7(\mathrm{CH}), 66.8\left(2 \mathrm{C}, \mathrm{CH}_{2}\right), 46.2\left(\mathrm{CH}_{2}\right), 42.4\left(\mathrm{CH}_{2}\right) ;{ }^{19} \mathrm{~F} \mathrm{NMR}\left(\mathrm{CDCl}_{3}\right.$, $235 \mathrm{MHz}) \delta-62.7$; IR $\left(\mathrm{film} / \mathrm{cm}^{-1}\right)$ 3466, 2857, 1648, 1607, 1432, 1326, 1226, 1166, 1116, 1069; HRMS (ES ${ }^{+}$) cald for $\mathrm{C}_{20} \mathrm{H}_{18} \mathrm{~F}_{3} \mathrm{NO}_{2}:(\mathrm{M}+\mathrm{H})^{+}$361.12841. Found: 361.12678 .

Thiophene-2-sulfonic acid [(E)-3-(3',4'-dichloro-biphenyl-2-yl)-acryloyl]-amide (11): Isolated in a yield of 68\% $\left(60 \%\right.$ over three steps) as a colourless oil. ${ }^{1} \mathrm{H}$ NMR $\left(\mathrm{CDCl}_{3}, 360 \mathrm{MHz}\right) \delta 8.79(\mathrm{bs}, 1 \mathrm{H}, \mathrm{NH}), 7.92(\mathrm{dd}, 1 \mathrm{H}, \mathrm{ArH}, \mathrm{J}=$ 1.4, $3.9 \mathrm{~Hz}), 7.69(\mathrm{dd}, 1 \mathrm{H}, \mathrm{ArH}, \mathrm{J}=1.4,5.0 \mathrm{~Hz}), 7.69(\mathrm{~d}, 1 \mathrm{H}, \mathrm{CH}=\mathrm{CH}, \mathrm{J}=15.6 \mathrm{~Hz}), 7.65(\mathrm{~m}, 1 \mathrm{H}, \operatorname{Ar} H), 7.48-$ $7.37(\mathrm{~m}, 3 \mathrm{H}, \operatorname{ArH}), 7.36(\mathrm{~d}, 1 \mathrm{H}, \operatorname{ArH}, \mathrm{J}=2.0 \mathrm{~Hz}), 7.30(\mathrm{~m}, 1 \mathrm{H}, \operatorname{ArH}), 7.11(\mathrm{dd}, 1 \mathrm{H}, \operatorname{ArH}, \mathrm{J}=3.9,5.0 \mathrm{~Hz}), 7.06(\mathrm{dd}$, $1 \mathrm{H}, \mathrm{ArH}, \mathrm{J}=2.1,8.2 \mathrm{~Hz}), 6.43(\mathrm{~d}, 1 \mathrm{H}, \mathrm{CH}=\mathrm{CH}, \mathrm{J}=15.5 \mathrm{~Hz}) ;{ }^{13} \mathrm{C} \mathrm{NMR}\left(\mathrm{CDCl}_{3}, 90.55 \mathrm{MHz}\right) \delta 162.7$ (quat), 144.4 $(\mathrm{CH}), 140.9$ (quat), 139.5 (quat), 138.7 (quat), $135.3(\mathrm{CH}), 134.2(\mathrm{CH}), 132.7$ (quat), 132.3 (quat), 131.7 (quat), 131.2 (CH), $130.7(\mathrm{CH}), 130.4(\mathrm{CH}), 130.4(\mathrm{CH}), 129.2(\mathrm{CH}), 128.5(\mathrm{CH}), 127.5(\mathrm{CH}), 127.1(\mathrm{CH}), 118.7(\mathrm{CH})$; IR $\left(\right.$ film $\left./ \mathrm{cm}^{-1}\right) 3244,1684,1623,1445,1132$.

(E)-3-(6-Methoxy-4'-trifluoromethyl-biphenyl-2-yl)-acrylic acid tert-butyl ester (19): Isolated in a yield of $68 \%$ as a yellow oil. ${ }^{1} \mathrm{H}$ NMR $\left(\mathrm{CDCl}_{3}, 360 \mathrm{MHz}\right) \delta 7.67(\mathrm{~d}, 2 \mathrm{H}, \mathrm{ArH}, \mathrm{J}=8.0 \mathrm{~Hz}), 7.47-7.33(\mathrm{~m}, 4 \mathrm{H}, \mathrm{CH}=\mathrm{CH}$, $\operatorname{Ar} H), 6.99(\mathrm{~d}, 1 \mathrm{H}, \operatorname{Ar} H, \mathrm{~J}=8.2 \mathrm{~Hz}), 6.90(\mathrm{dd}, 1 \mathrm{H}, \operatorname{Ar} H, \mathrm{~J}=7.6,1.1 \mathrm{~Hz}), 6.55(\mathrm{~d}, 1 \mathrm{H}, \mathrm{CH}=\mathrm{CH}, \mathrm{J}=16.1 \mathrm{~Hz}), 3.95$ (s, $\left.3 \mathrm{H}, \mathrm{OCH}_{3}\right), 1.43\left(\mathrm{~s}, 9 \mathrm{H}, \mathrm{CCH}_{3}\right) ;{ }^{13} \mathrm{C} \mathrm{NMR}\left(\mathrm{CDCl}_{3}, 90.55 \mathrm{MHz}\right) \delta 167.0$ (quat), 159.3 (quat), 144.1 (quat), 143.7 (quat), $137.9(\mathrm{CH}), 130.2(2 \mathrm{C}, \mathrm{CH}), 129.9(\mathrm{CH}), 125.1(\mathrm{q}, 2 \mathrm{C}, \mathrm{CH}, \mathrm{J}=3.6 \mathrm{~Hz}), 124.9(\mathrm{CH}), 122.6(\mathrm{CH}), 121.5$ (quat), $110.6(\mathrm{CH}), 80.0$ (quat), $55.7\left(\mathrm{CH}_{3}\right), 28.1\left(3 \mathrm{C}, \mathrm{CH}_{3}\right) ;{ }^{19} \mathrm{~F} \mathrm{NMR}\left(\mathrm{CDCl}_{3}, 235 \mathrm{MHz}\right) \delta-63.2 ; \mathrm{IR}\left(\mathrm{film} / \mathrm{cm}^{-1}\right)$ 2976, 1702, 1624, 1467, 1325, 1257, 1151, 1123; HRMS $\left(\mathrm{ES}^{+}\right)$cald for $\mathrm{C}_{21} \mathrm{H}_{21} \mathrm{~F}_{3} \mathrm{O}_{3}:(\mathrm{M}+\mathrm{H})^{+}$378.14373. Found: 378.14259 .

(E)-3-(5,6-Dimethoxy-4'-trifluoromethyl-biphenyl-2-yl)-acrylic acid tert-butyl ester (20): Isolated in a yield of $32 \%$ as a slightly yellow oil. ${ }^{1} \mathrm{H} \mathrm{NMR}\left(\mathrm{CDCl}_{3}, 360 \mathrm{MHz}\right) \delta 7.65(\mathrm{~d}, 2 \mathrm{H}, \mathrm{ArH}, \mathrm{J}=8.0 \mathrm{~Hz}), 7.41(\mathrm{~d}, 1 \mathrm{H}, \mathrm{CH}=\mathrm{CH}, \mathrm{J}$ $=16.2 \mathrm{~Hz}), 7.39(\mathrm{~d}, 2 \mathrm{H}, \mathrm{ArH}, \mathrm{J}=7.9 \mathrm{~Hz}), 7.00(\mathrm{q}, 2 \mathrm{H}, \operatorname{ArH}, \mathrm{J}=8.5 \mathrm{~Hz}), 6.45(\mathrm{~d}, 1 \mathrm{H}, \mathrm{CH}=\mathrm{CH}, \mathrm{J}=16.2 \mathrm{~Hz}), 3.94$ $\left(\mathrm{s}, 3 \mathrm{H}, \mathrm{OCH}_{3}\right), 3.85\left(\mathrm{~s}, 3 \mathrm{H}, \mathrm{OCH}_{3}\right), 1.44\left(\mathrm{~s}, 9 \mathrm{H}, \mathrm{CCH}_{3}\right) ;{ }^{13} \mathrm{C} \mathrm{NMR}\left(\mathrm{CDCl}_{3}, 90.55 \mathrm{MHz}\right) \delta 166.6$ (quat), 152.8 (quat), 148.7 (quat), 144.1 (quat), $137.9(\mathrm{CH}), 134.8$ (quat), $130.2(2 \mathrm{C}, \mathrm{CH}), 129.2$ (d, quat, $\mathrm{J}=32.5 \mathrm{~Hz}$ ), 127.0 (quat), $125.8(\mathrm{CH}), 125.8(\mathrm{CH}), 125.1(\mathrm{q}, 2 \mathrm{C}, \mathrm{CH}, \mathrm{J}=3.6 \mathrm{~Hz}), 124.3\left(\mathrm{q}, \mathrm{CF}_{3}, \mathrm{~J}=289.4 \mathrm{~Hz}\right), 112.9(\mathrm{CH}), 80.3$ (quat), 60.2 $\left(\mathrm{CH}_{3}\right), 56.0\left(\mathrm{CH}_{3}\right), 28.1\left(3 \mathrm{C}, \mathrm{CH}_{3}\right) ;{ }^{19} \mathrm{~F} \mathrm{NMR}\left(\mathrm{CDCl}_{3}, 235 \mathrm{MHz}\right) \delta-63.7 ; \mathrm{IR}\left(\mathrm{film} / \mathrm{cm}^{-1}\right)$ 2937, 1706, 1479, 1325 , 1153, 1125; HRMS (ES $)$ cald for $\mathrm{C}_{22} \mathrm{H}_{23} \mathrm{~F}_{3} \mathrm{O}_{4}:(\mathrm{M}+\mathrm{H})^{+}$408.15430. Found: 408.15300. 
<smiles>COc1cccc(-c2ccccc2/C=C/C(=O)OC(C)(O)Cl)c1</smiles>

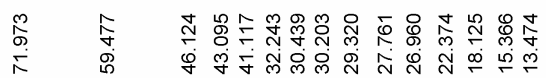

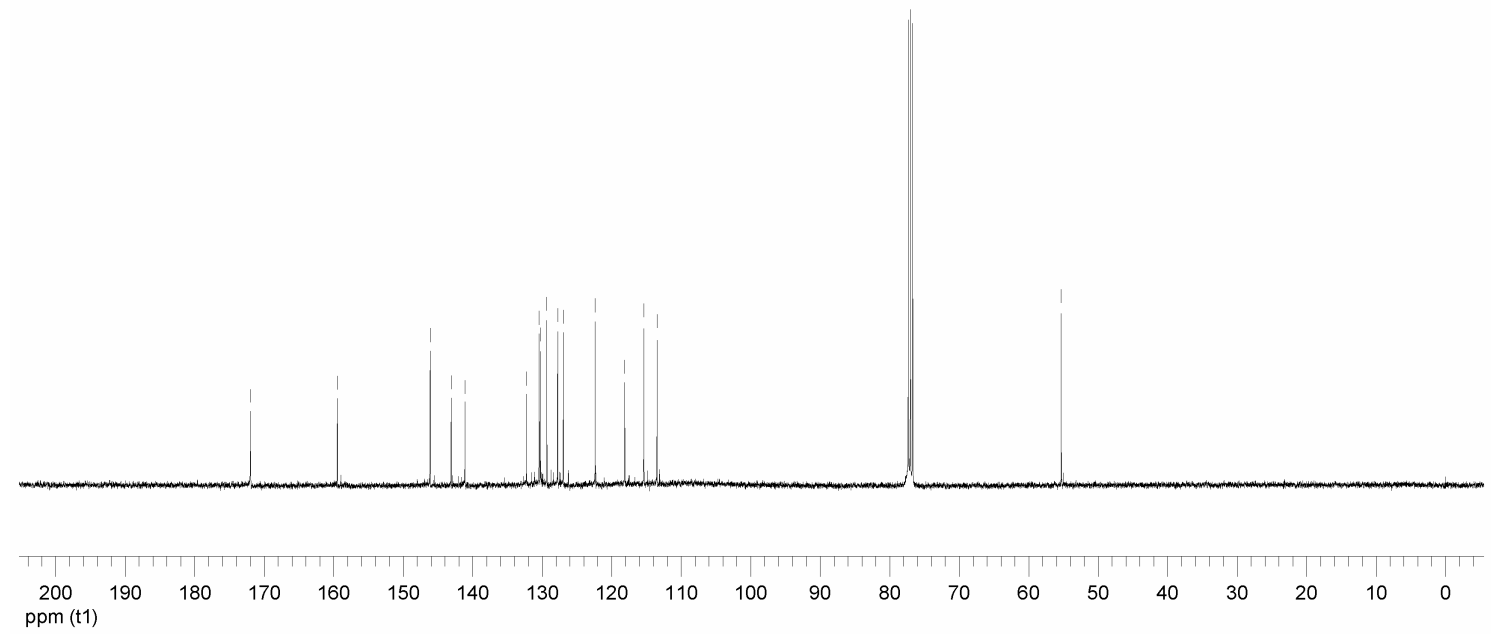


<smiles>CCCCOC(=O)/C=C/c1ccccc1-c1ccc(Br)cc1</smiles>
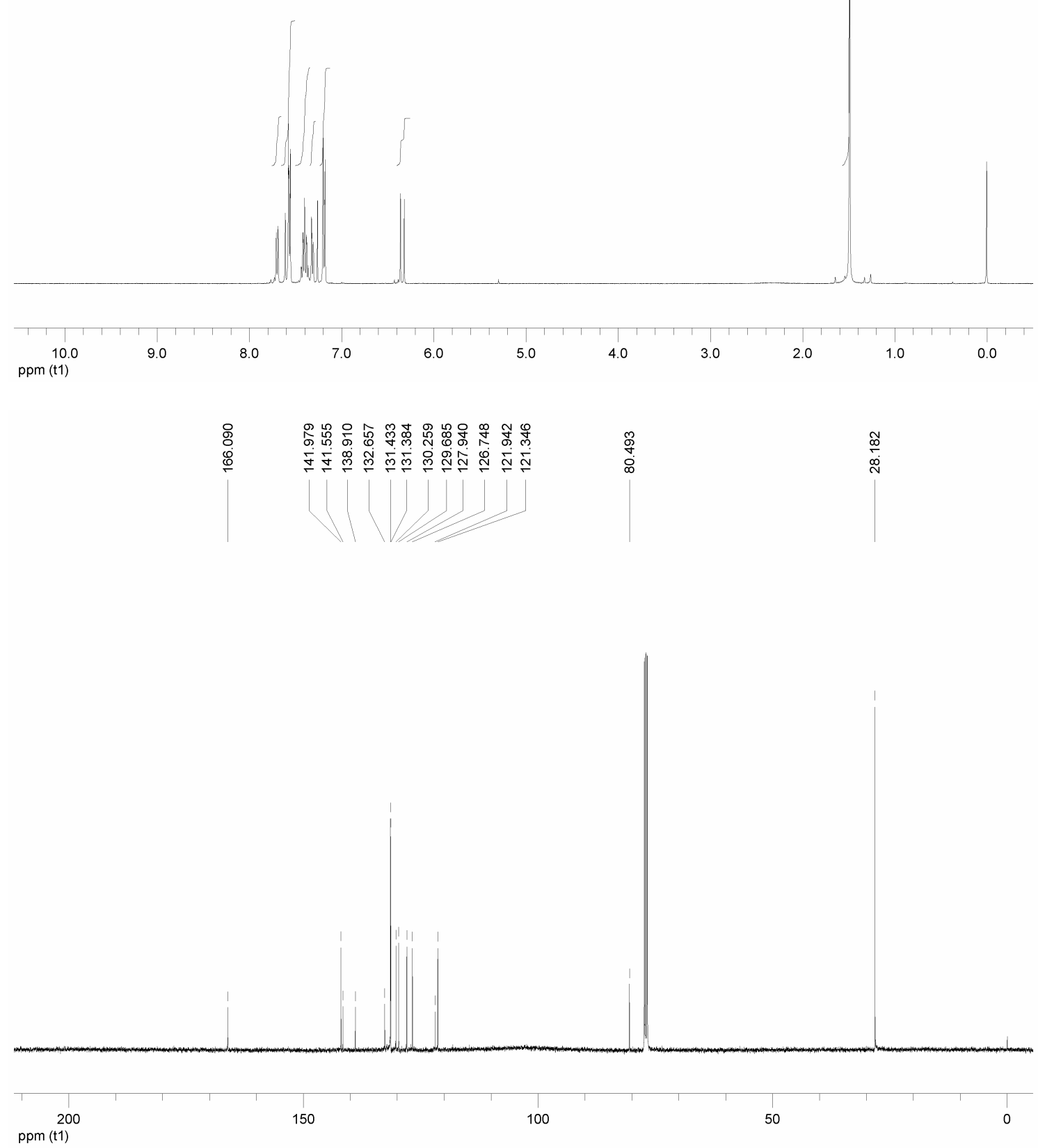

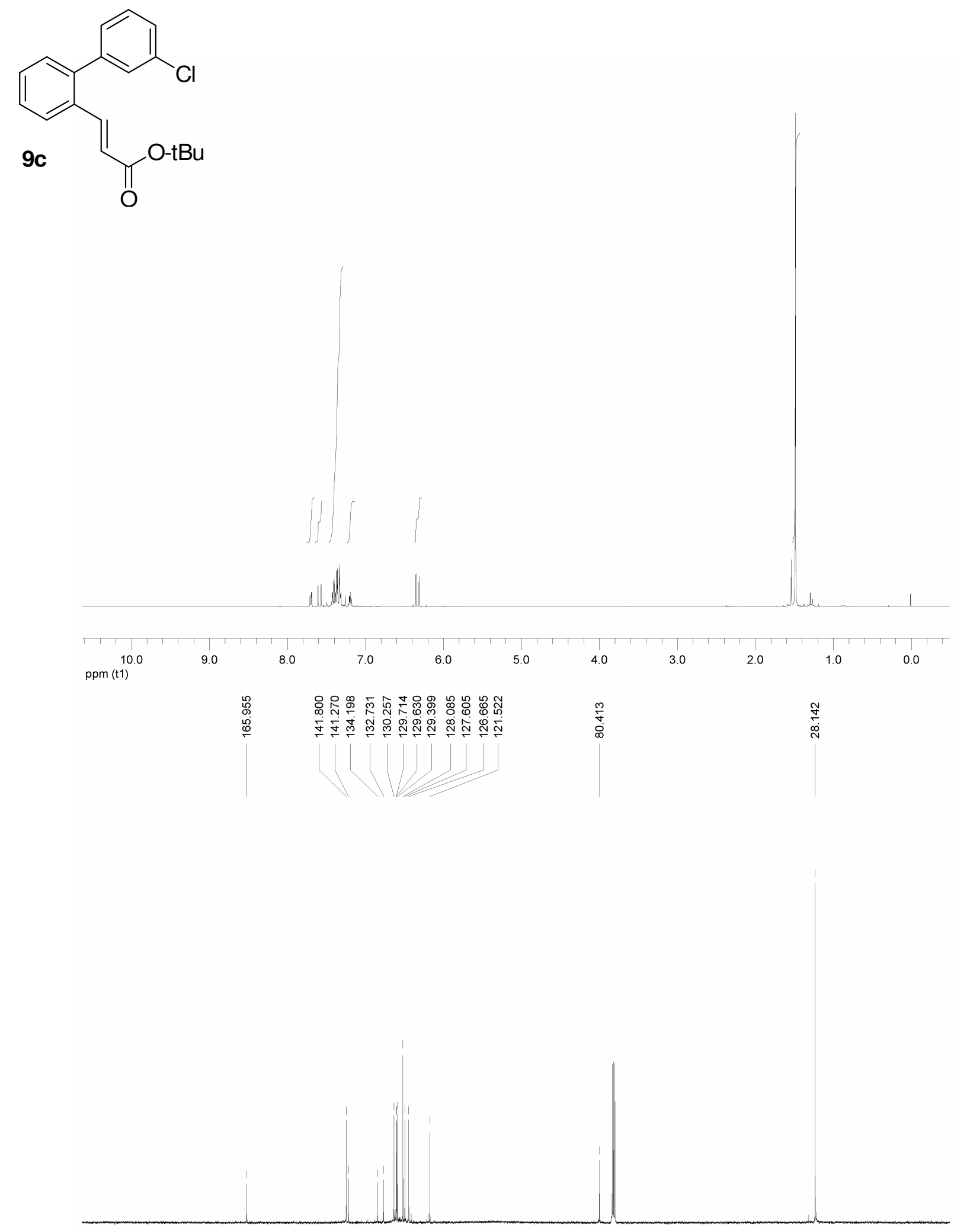

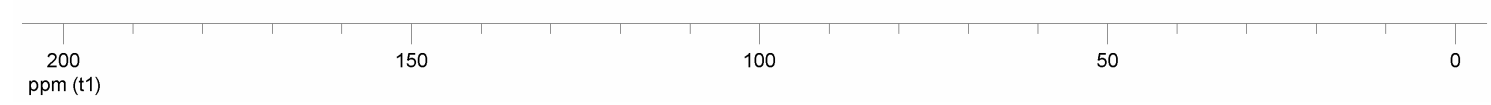


<smiles>CCCCOC(=O)/C=C/c1ccccc1-c1cccc(F)c1</smiles>

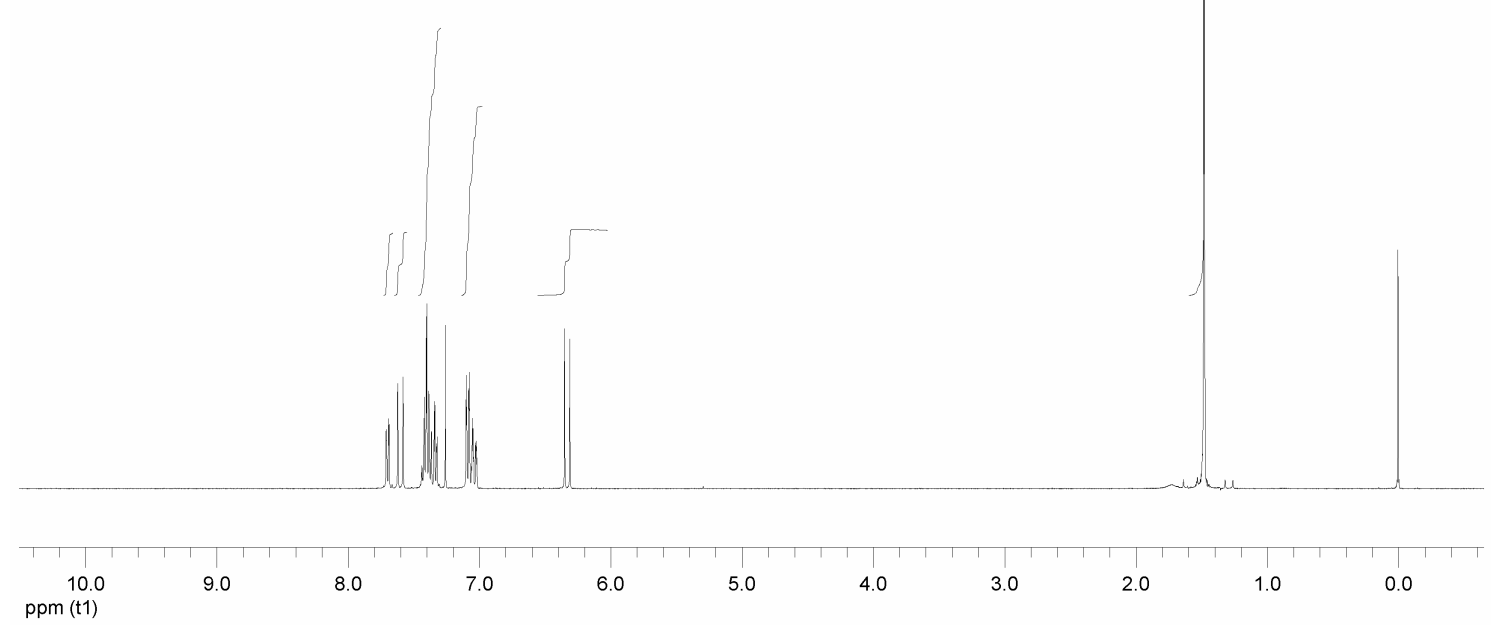

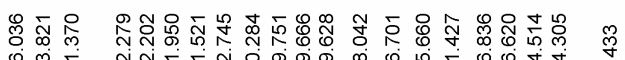

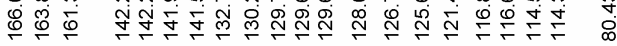

$\underset{\substack{\infty \\ \infty}}{\stackrel{\infty}{\infty}}$

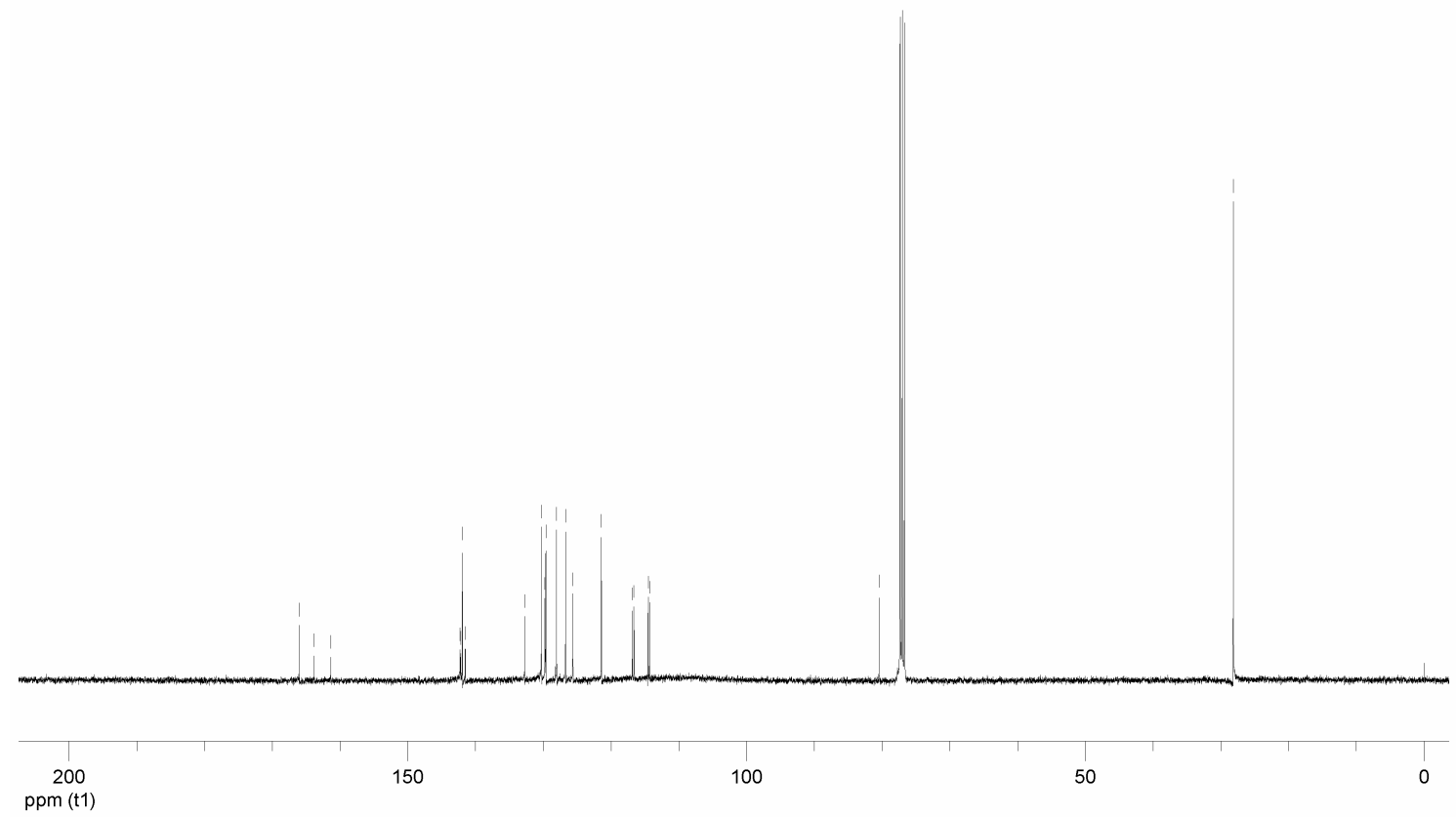


<smiles>CCCOC(=O)/C=C/c1ccccc1-c1ccccc1[N+](=O)[O-]</smiles>
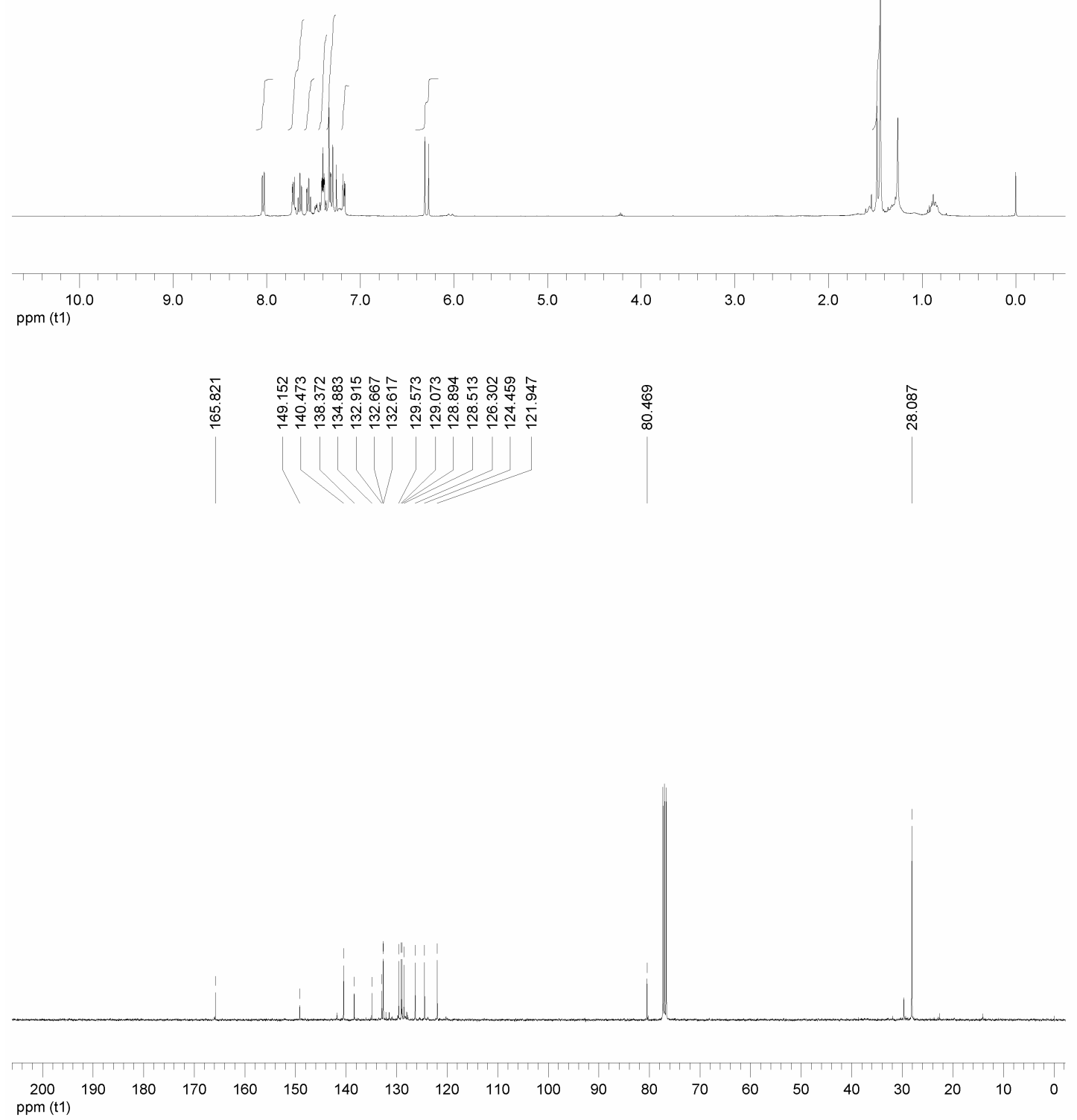

S12 

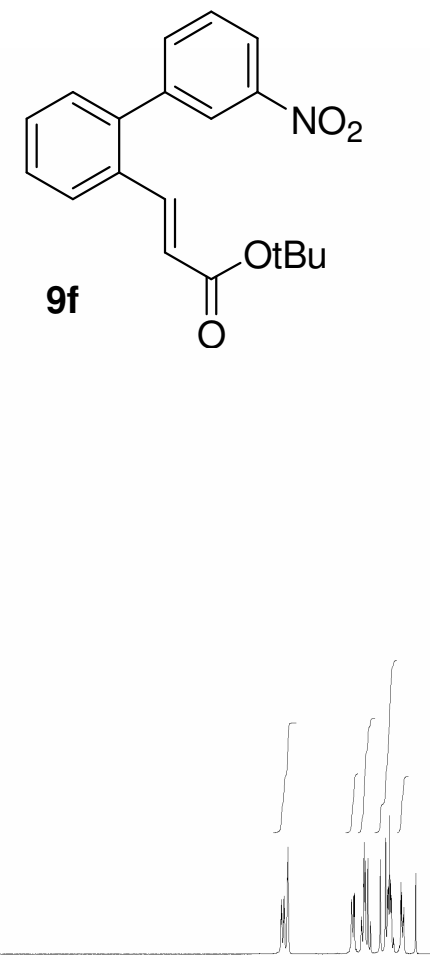

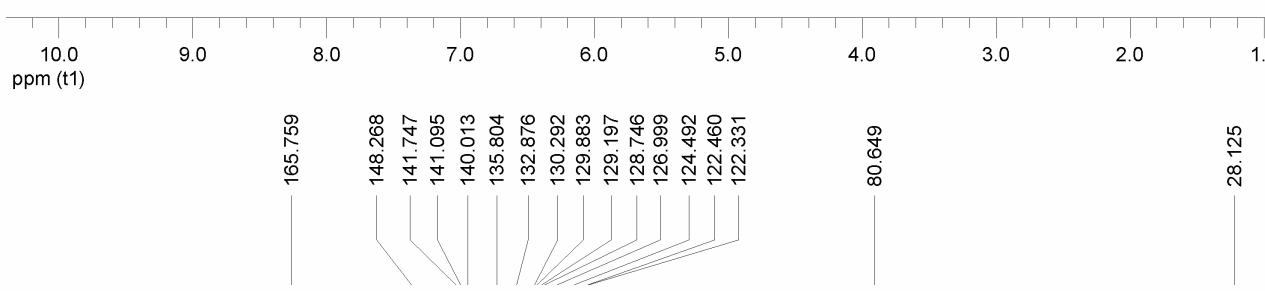

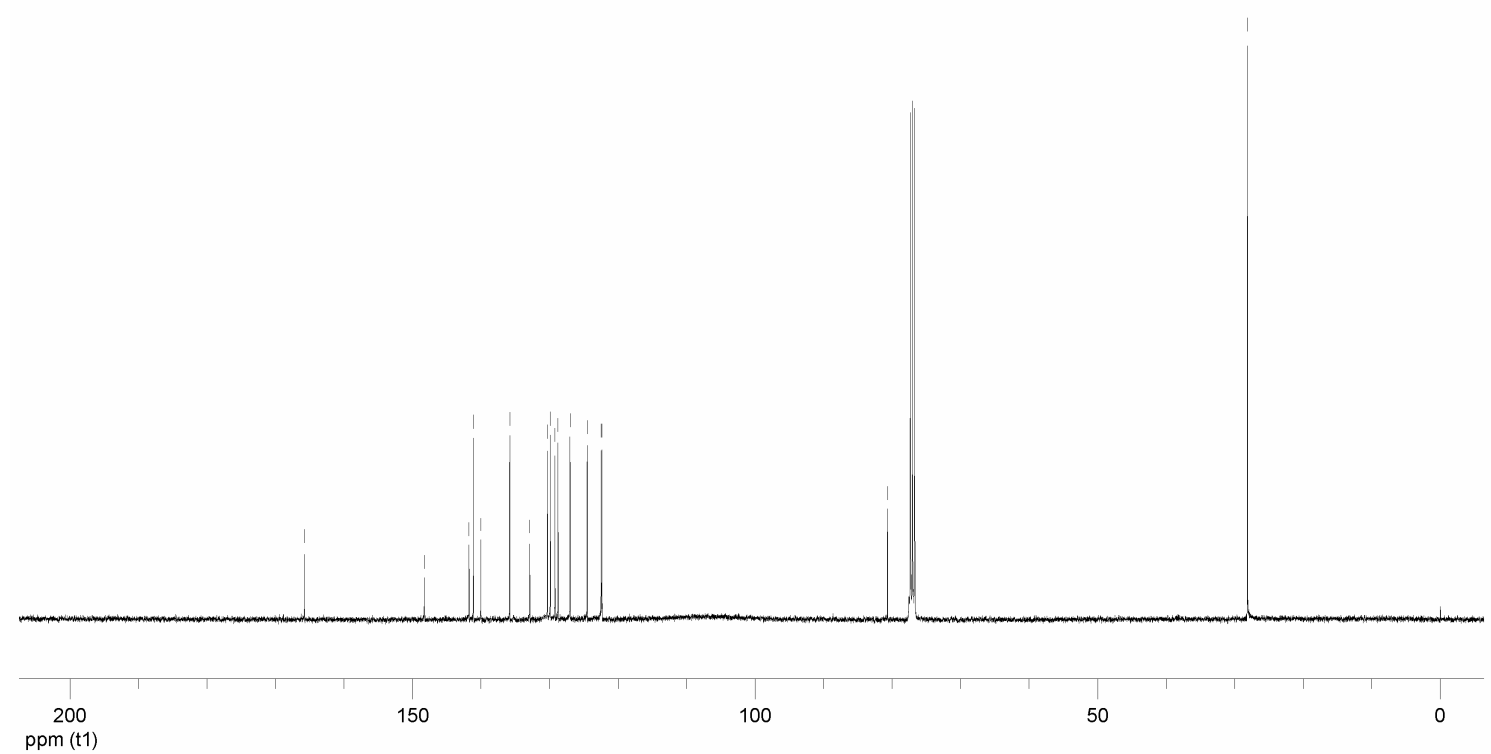



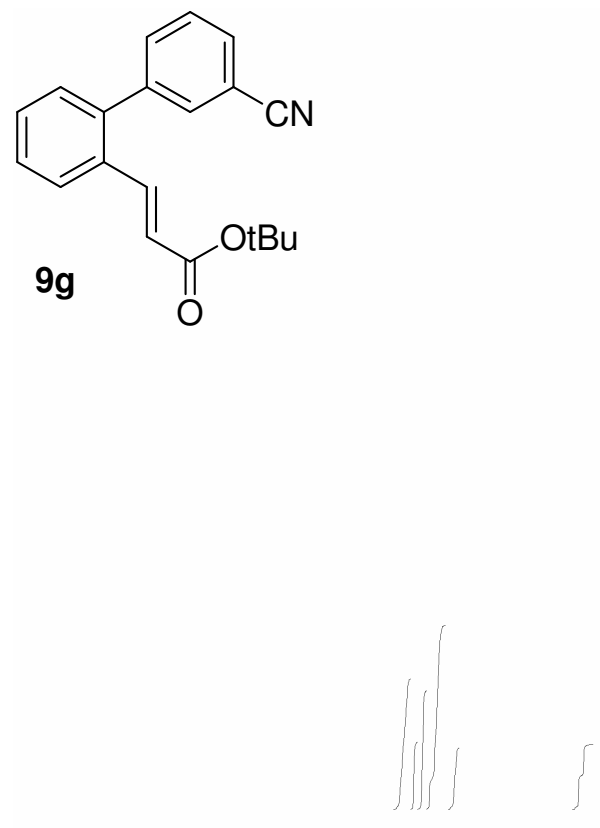

MUN W W

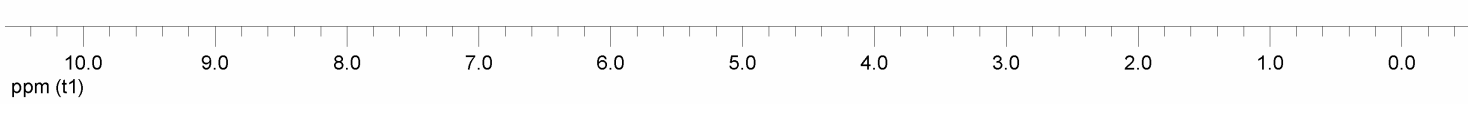

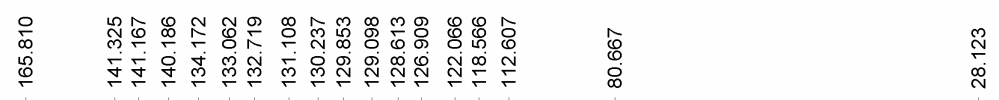

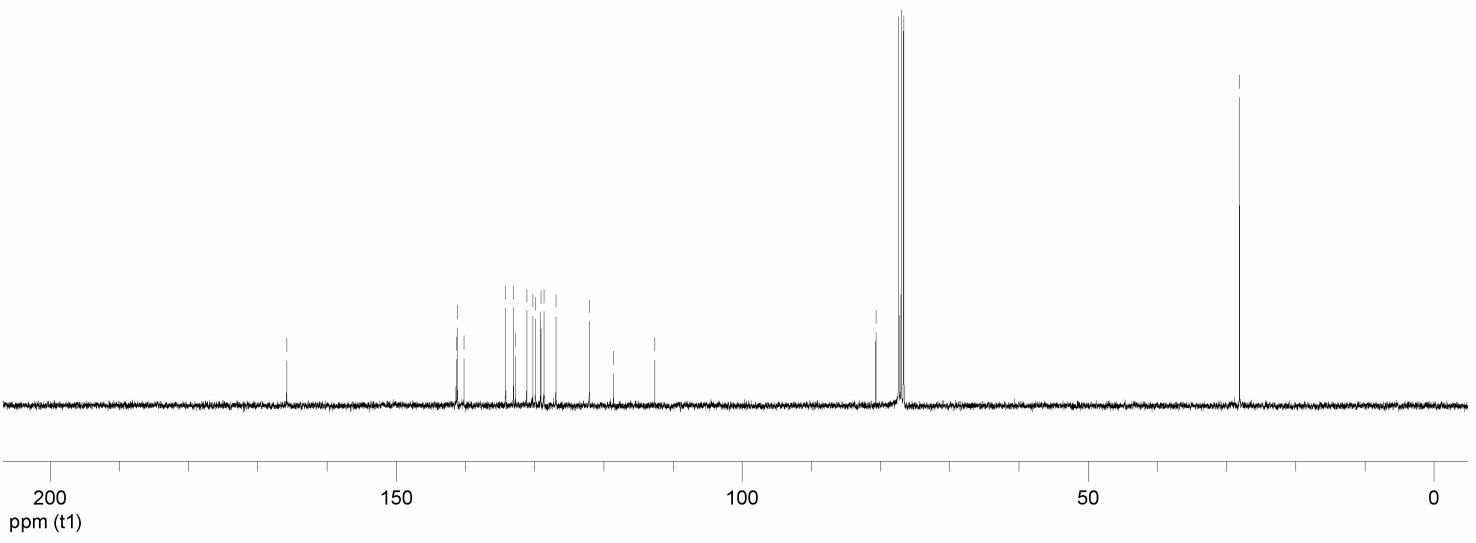



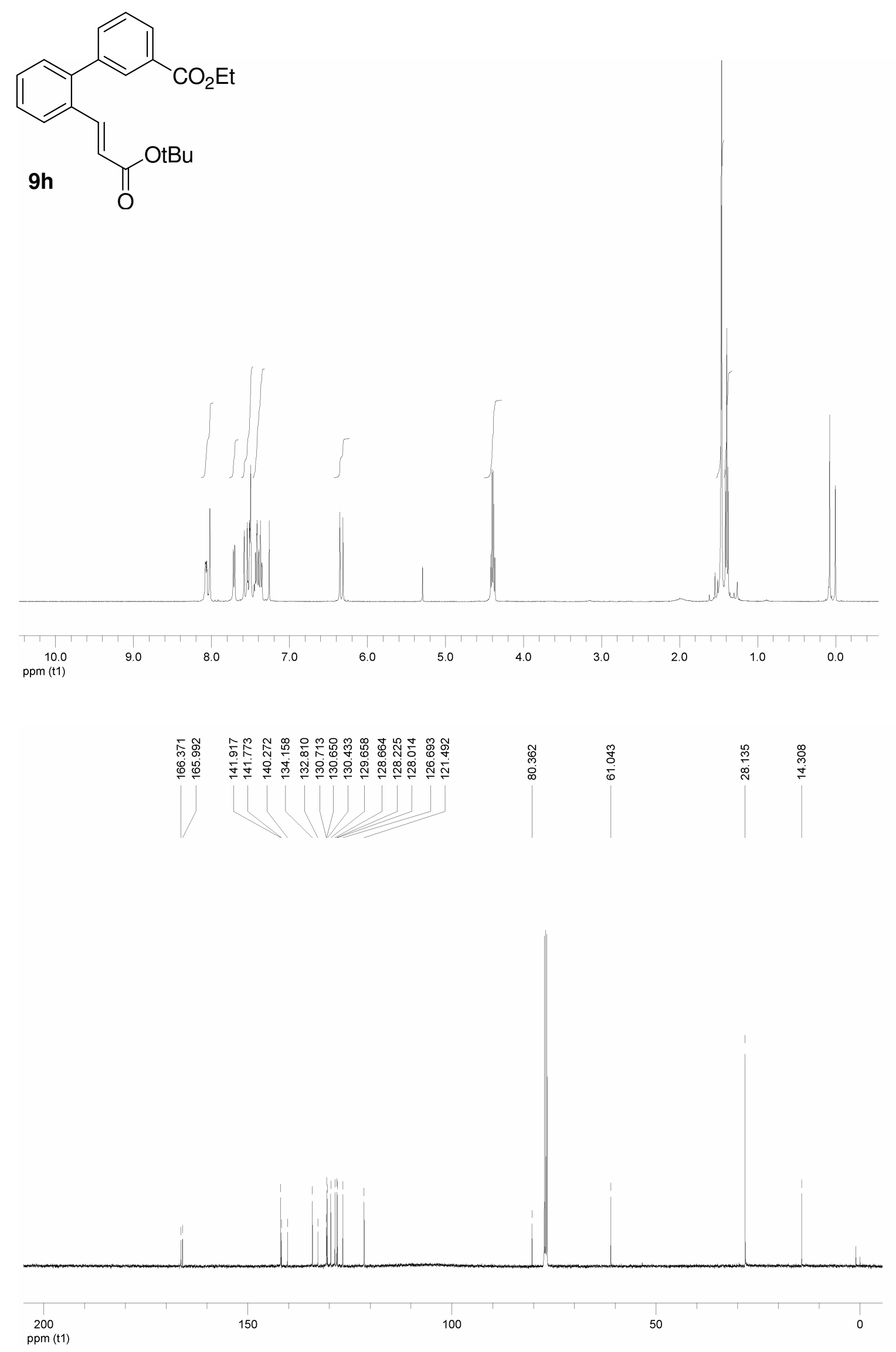
<smiles>CCCOC(=O)/C=C/c1ccccc1-c1ccc(C(F)(F)F)cc1</smiles><smiles>CC#CC</smiles>
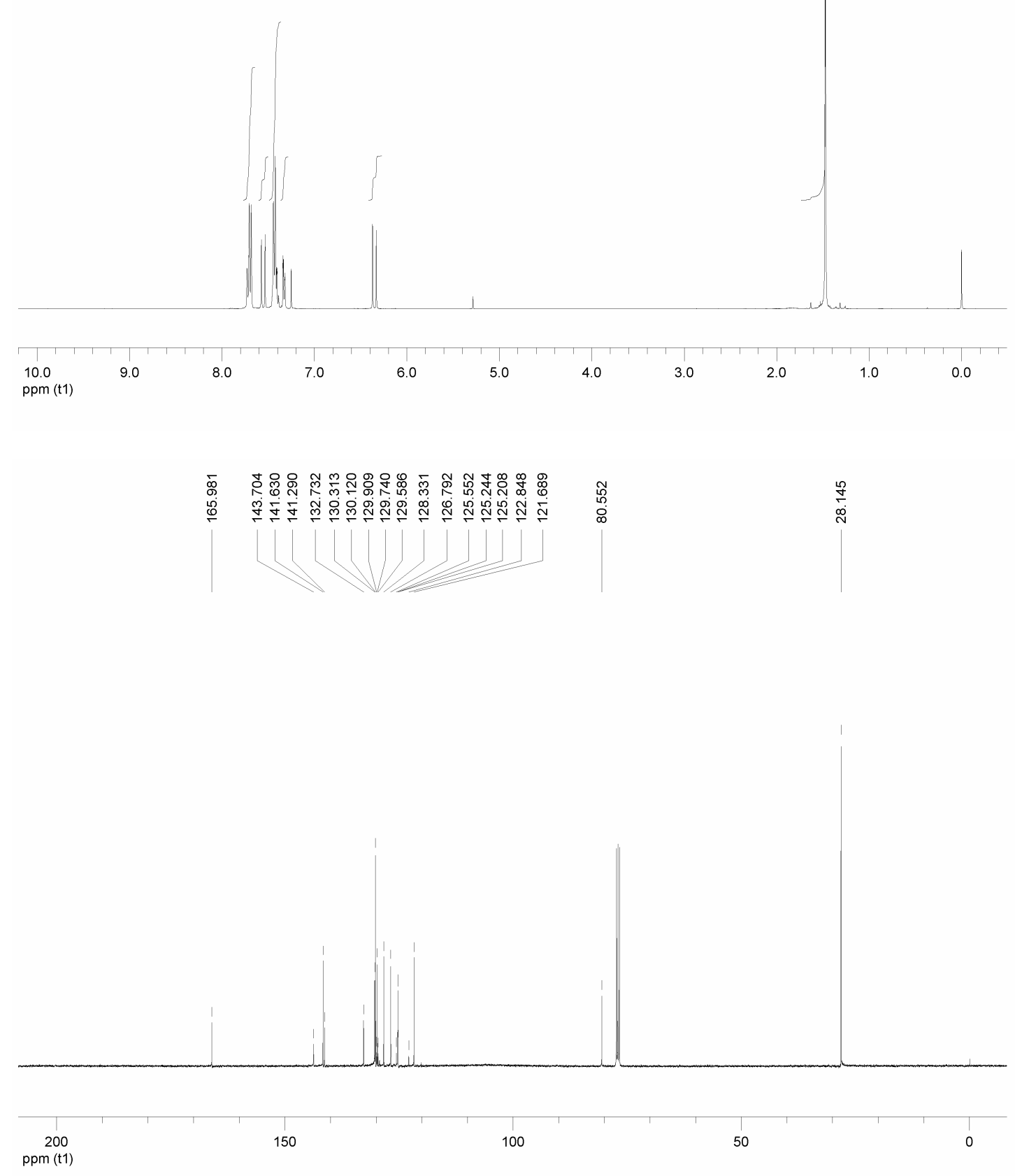
<smiles>CCCCOC(=O)/C=C/c1ccccc1-c1ccc(Cl)c(Cl)c1</smiles>
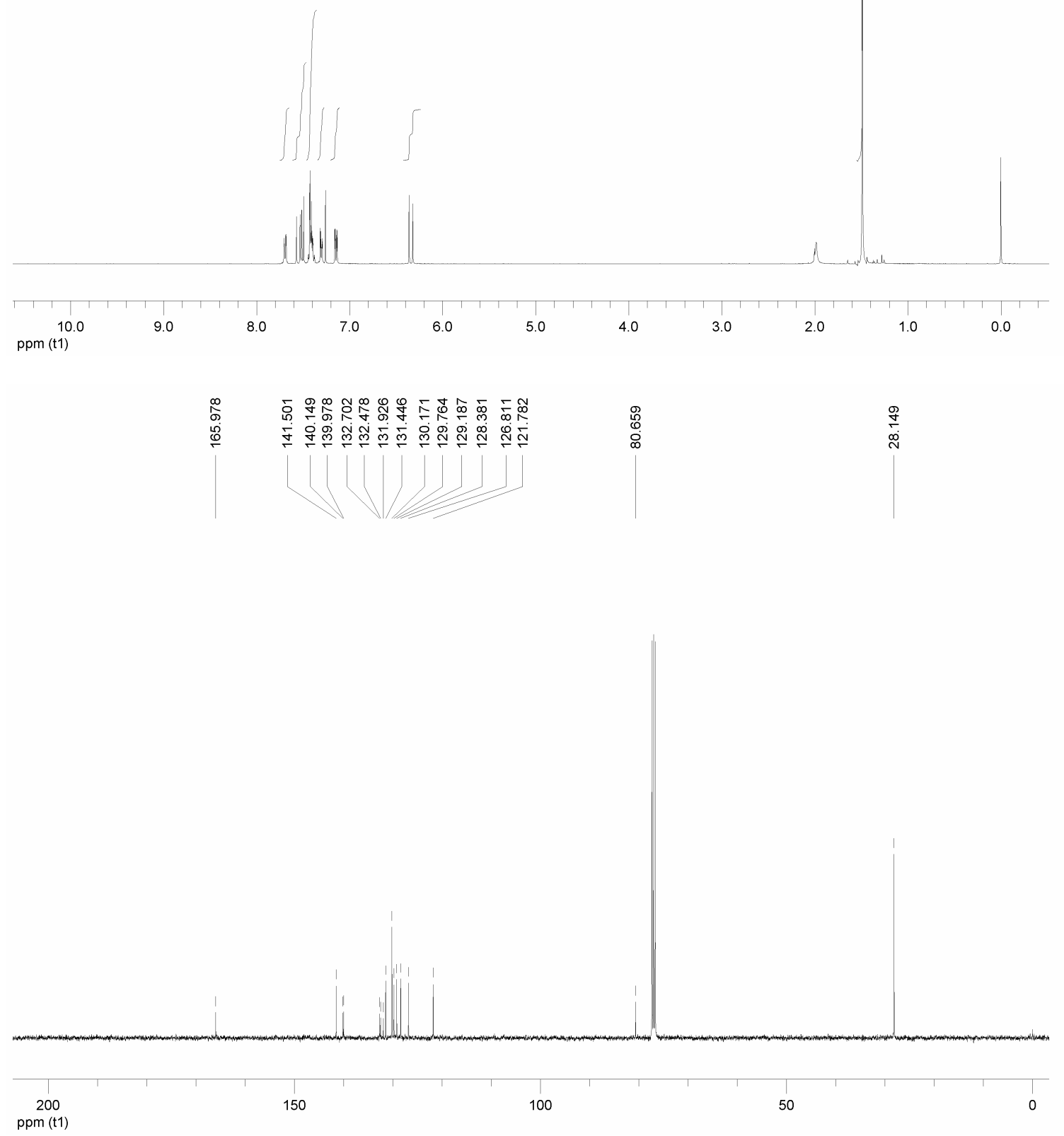
<smiles>CCCOC(=O)/C=C/c1ccccc1-c1ccc(Cl)cc1</smiles>

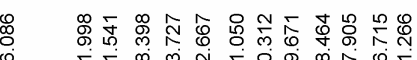

@

密

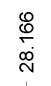

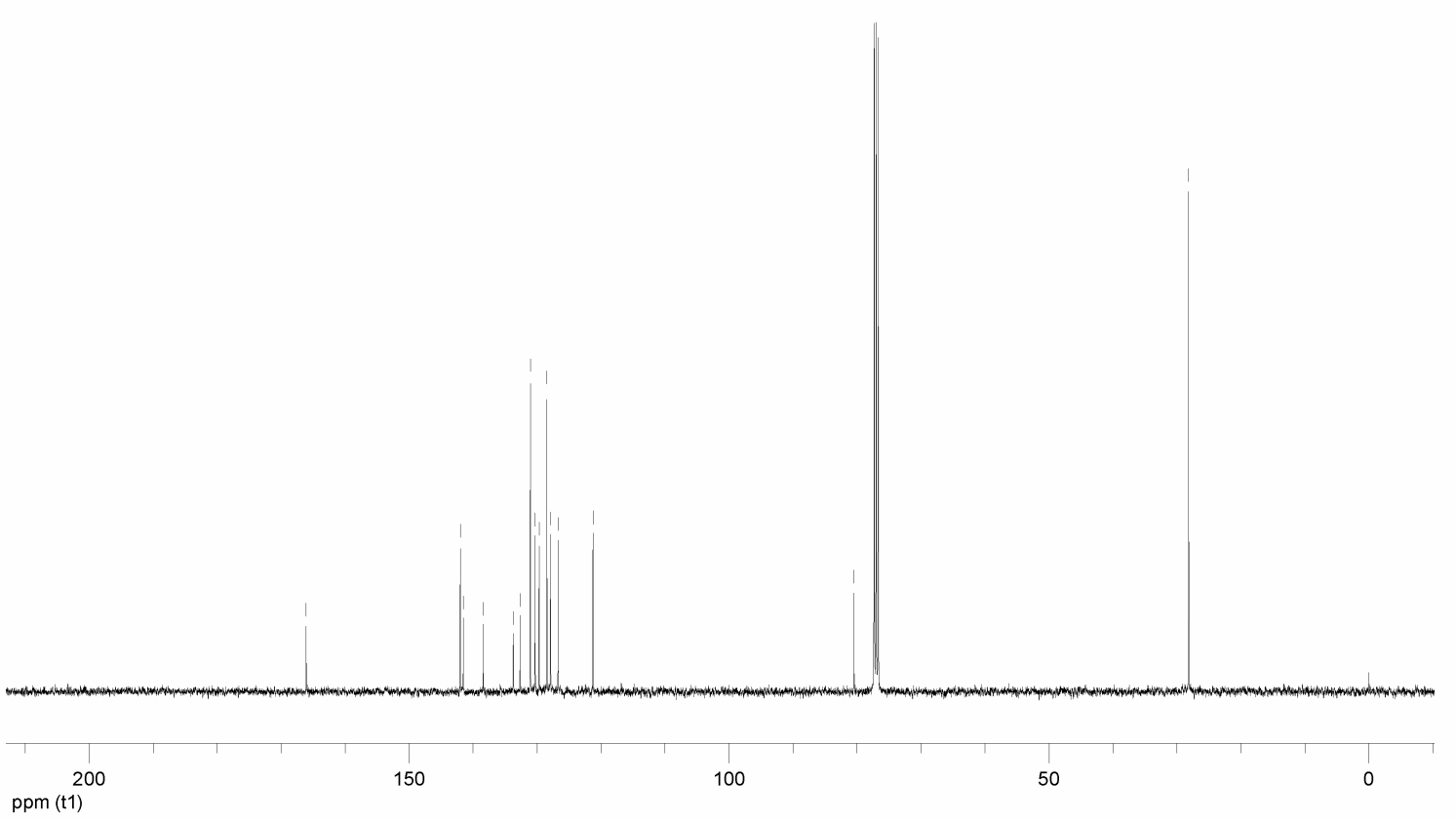


<smiles>CCCOC(=O)/C=C/c1ccccc1-c1cccs1</smiles>

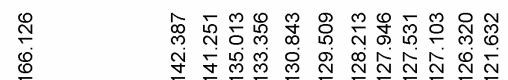

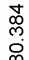

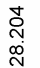

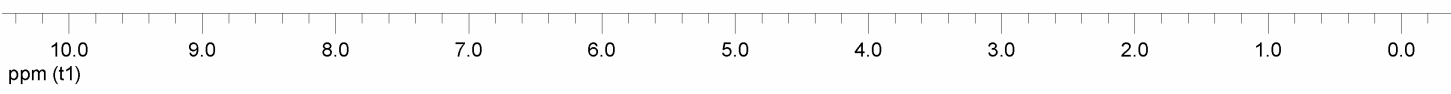

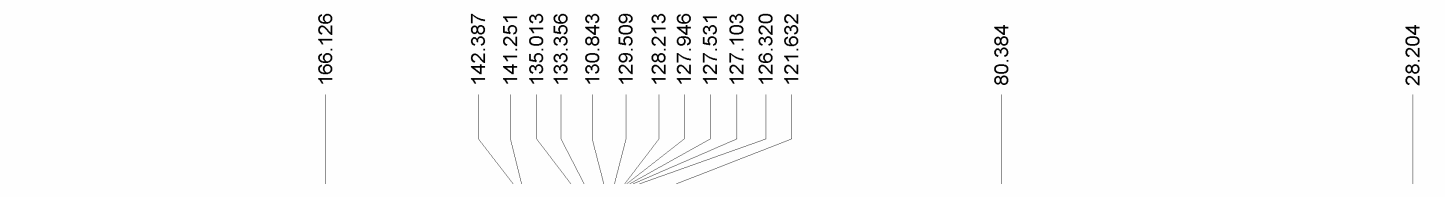

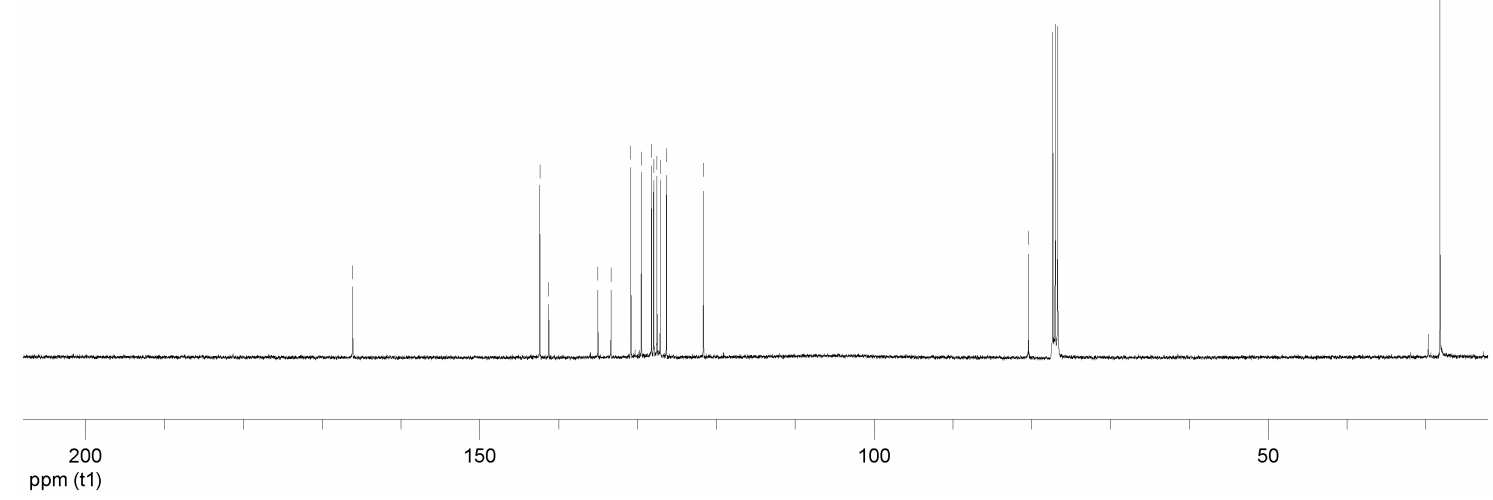


<smiles>CCCCOC(=O)/C=C/c1ccc(C)cc1-c1cccc(Cl)c1</smiles>

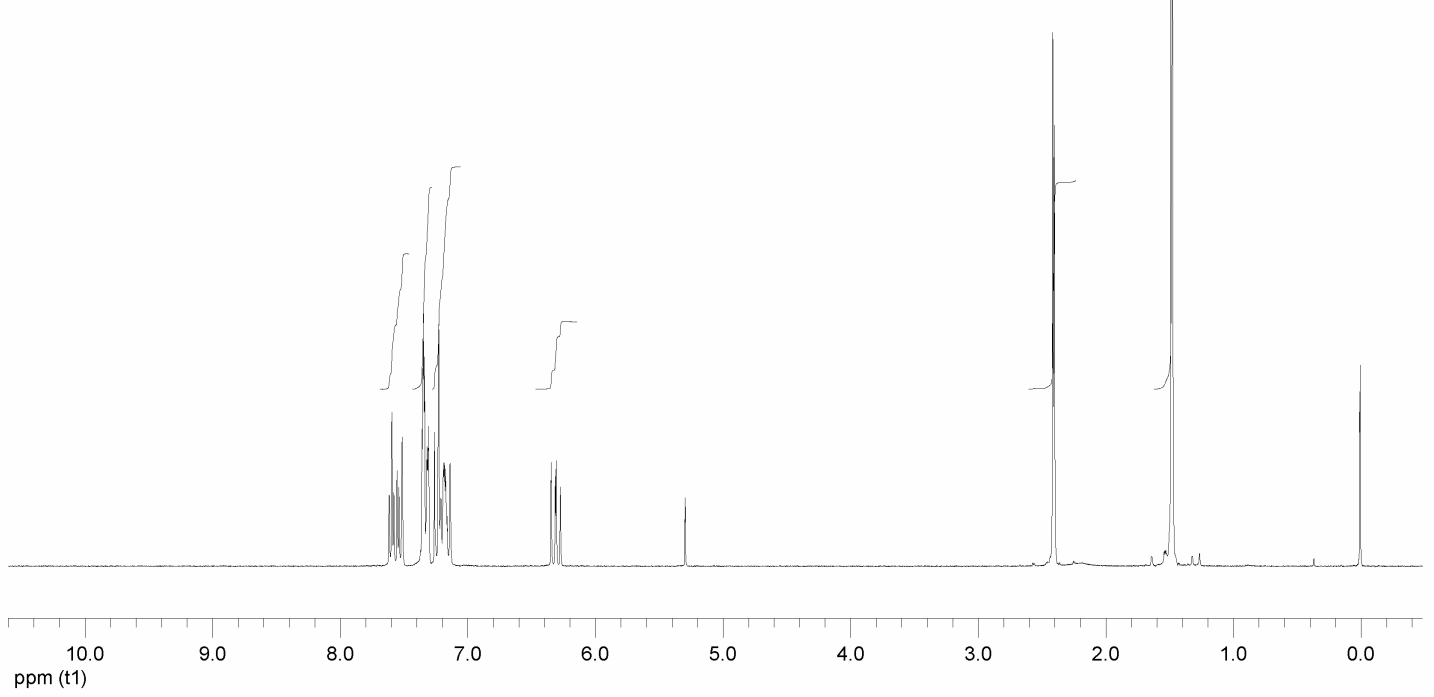

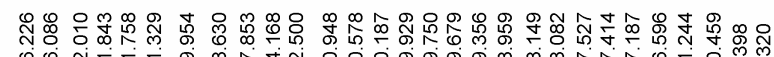

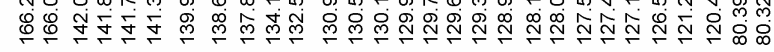

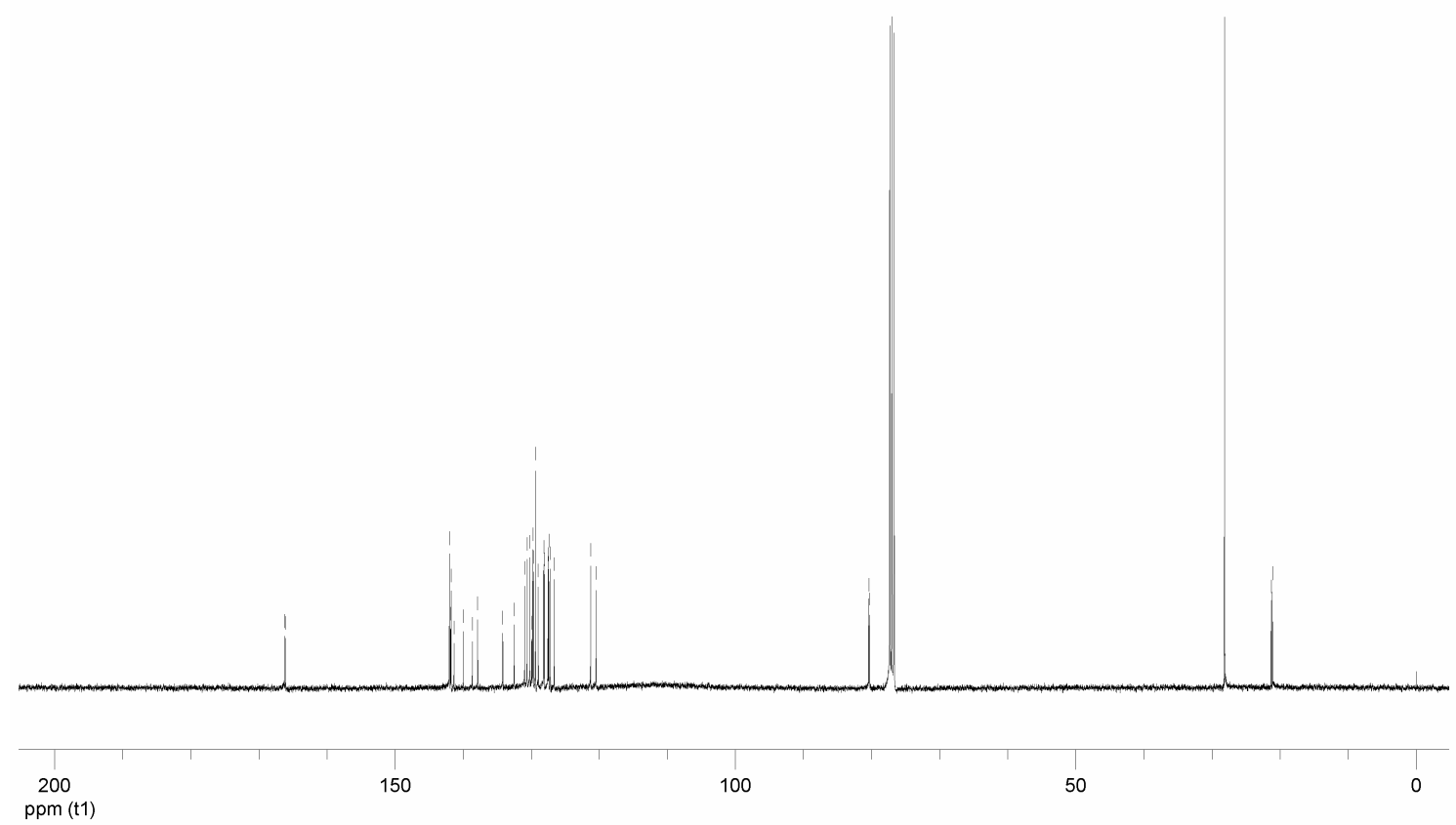


<smiles>CCCOC(=O)/C=C/c1c(C)ccc(C)c1-c1cccc(Cl)c1</smiles>

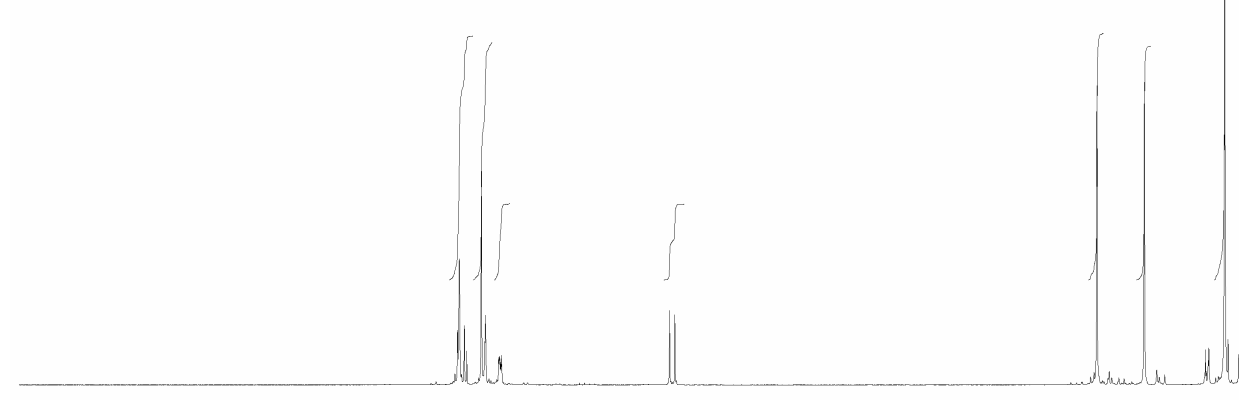

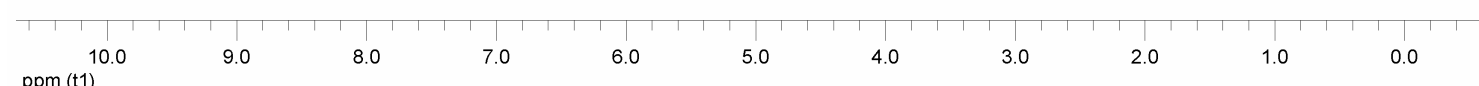

ppm (t1)

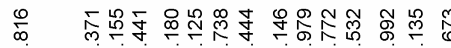

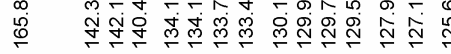

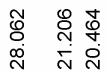

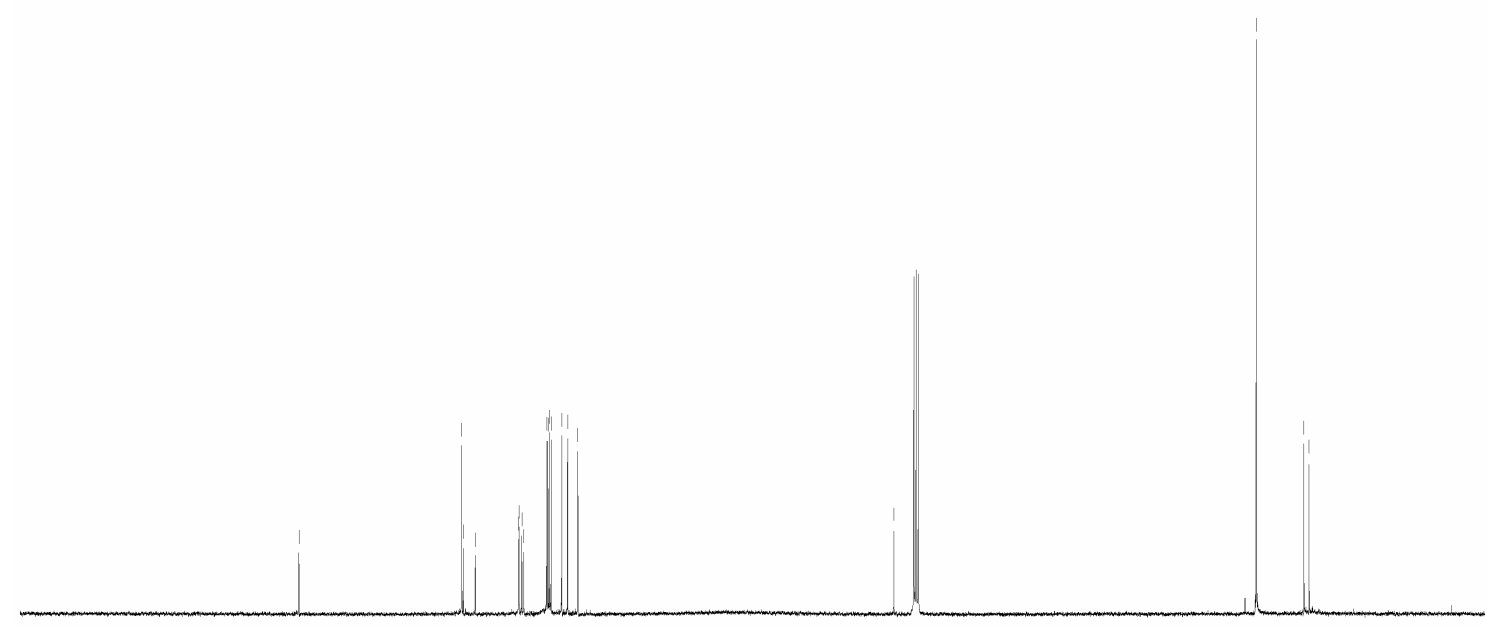
200
ppm (t1) 
<smiles>CCCOC(=O)/C=C/c1cc2c(cc1-c1ccc(C(F)(F)F)cc1)OCO2</smiles>

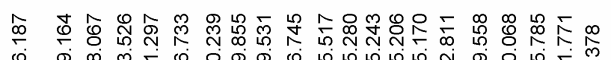

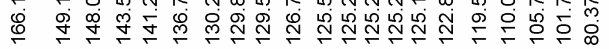

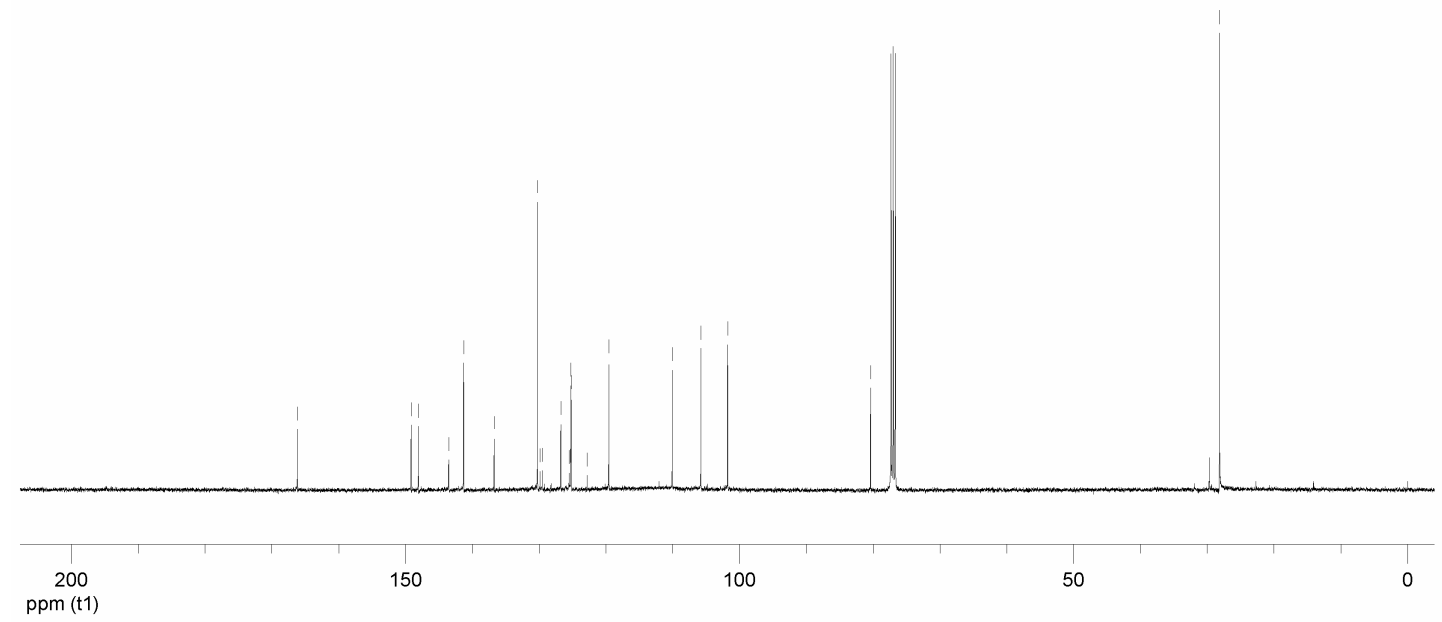



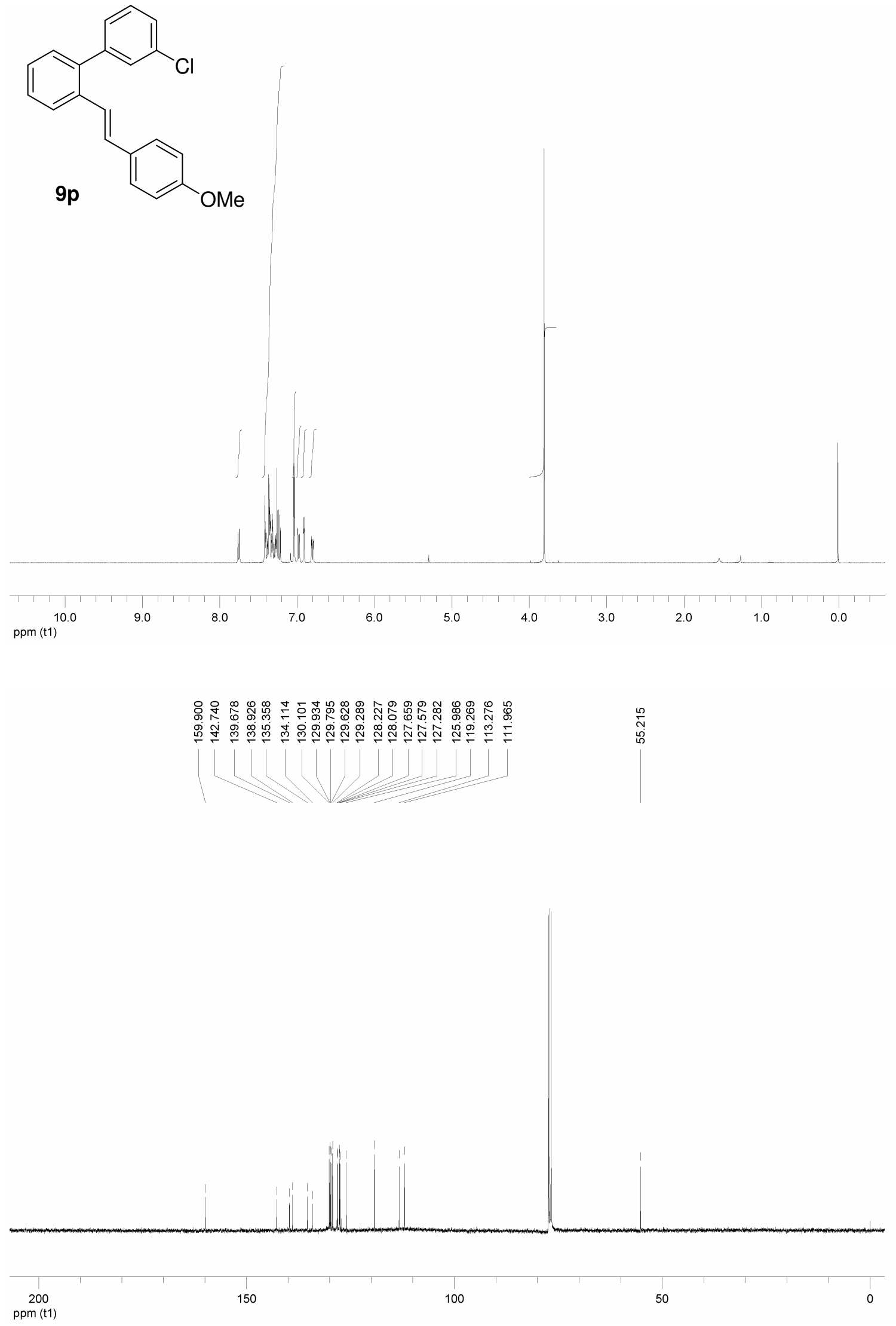

S23 


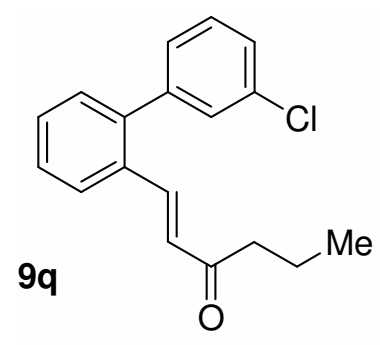

10.0

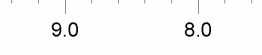

7.0
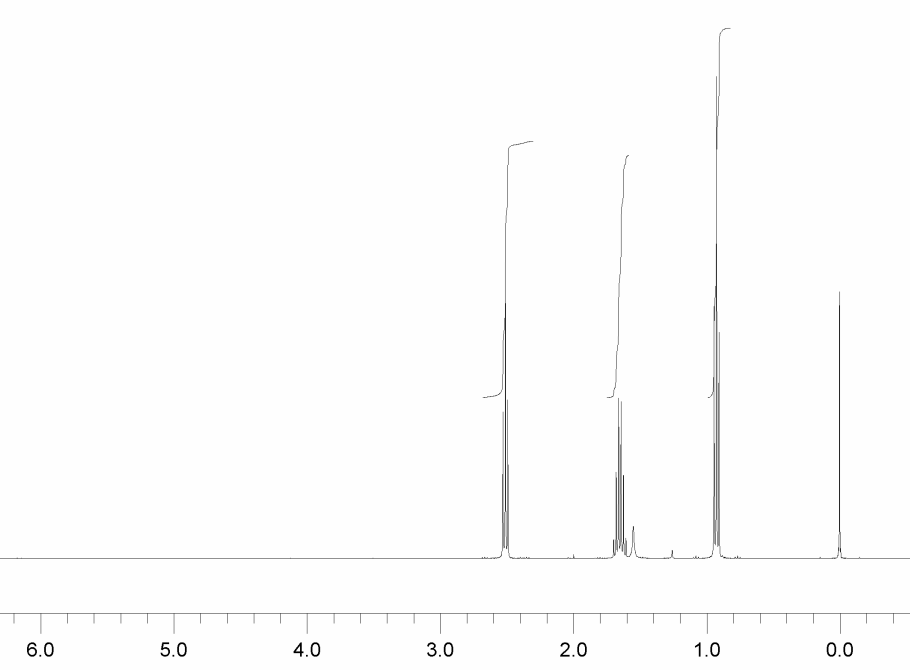

ppm (t1)

ֻ̊.

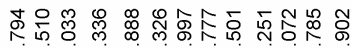

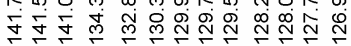

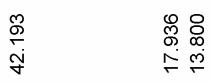

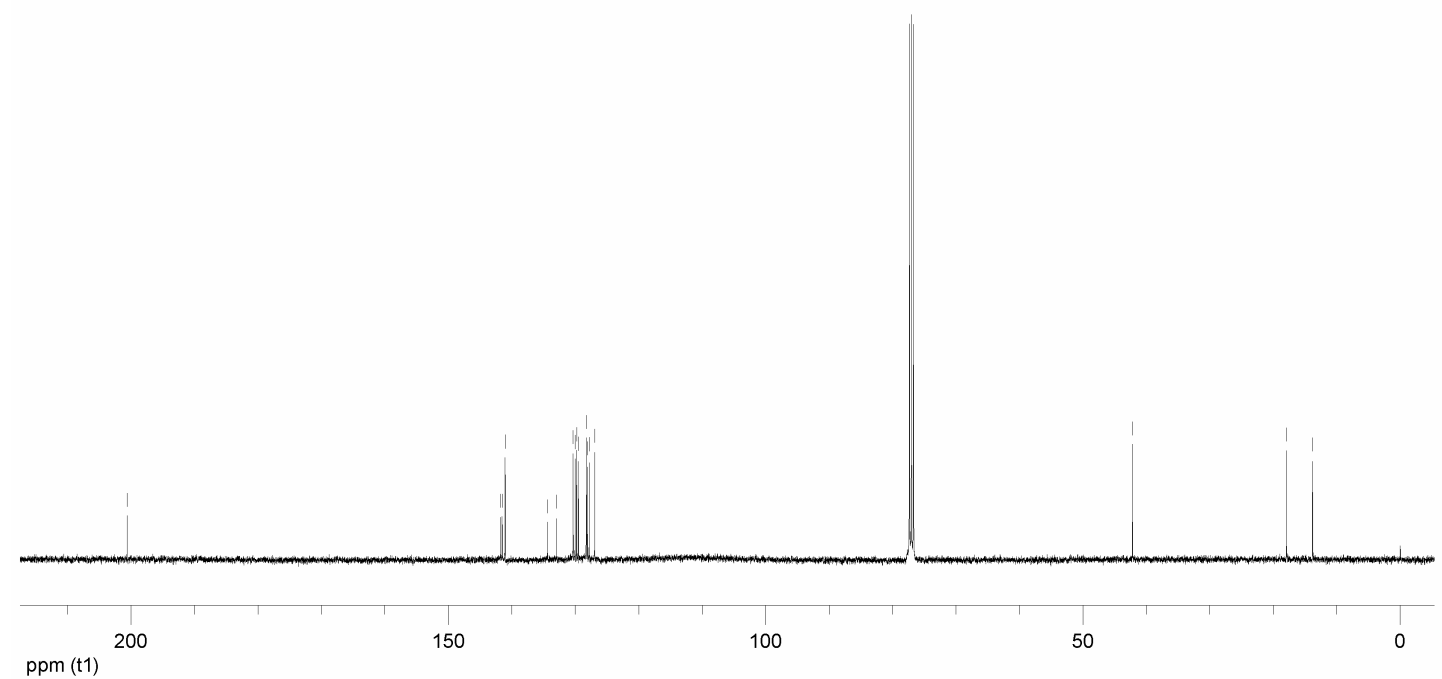


<smiles>CNC(=O)/C=C/c1ccccc1-c1cccc(Cl)c1</smiles>

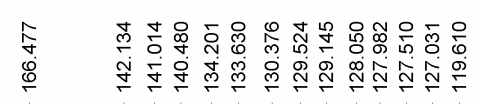

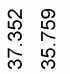

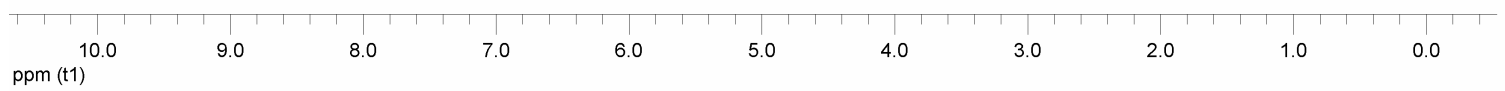

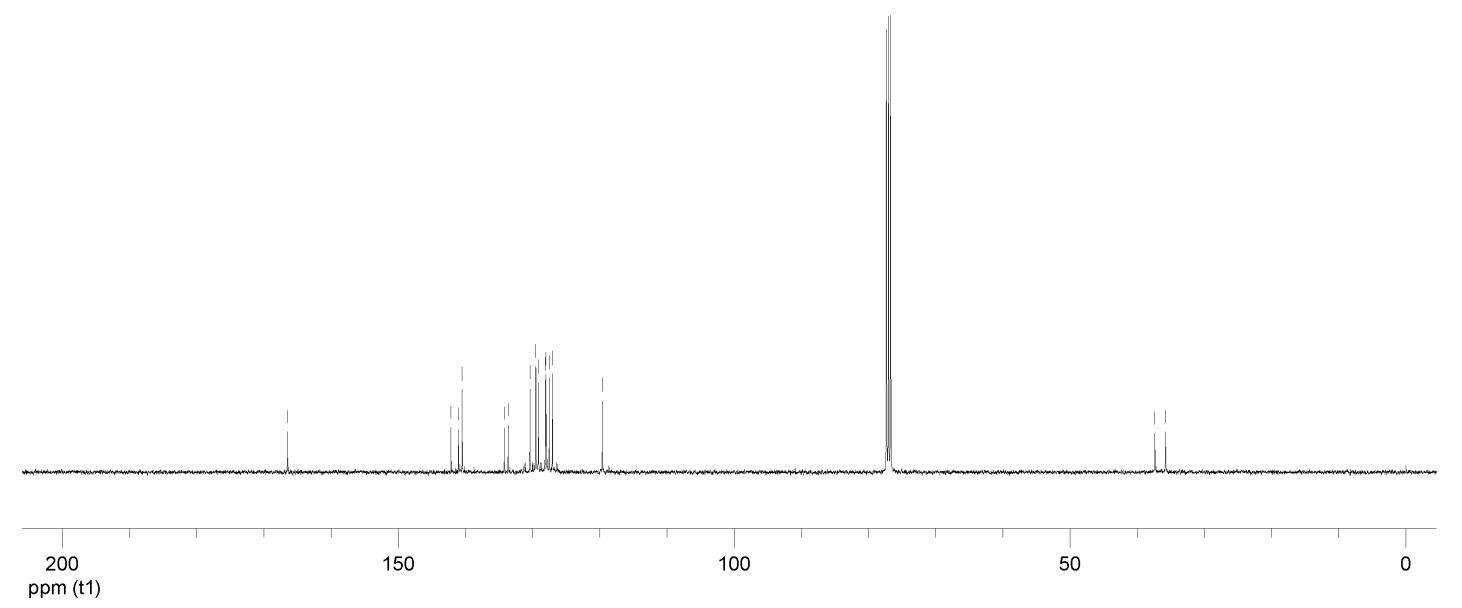


<smiles>O=C(/C=C/c1ccccc1-c1ccc(C(F)(F)F)cc1)N1CCOCC1</smiles>
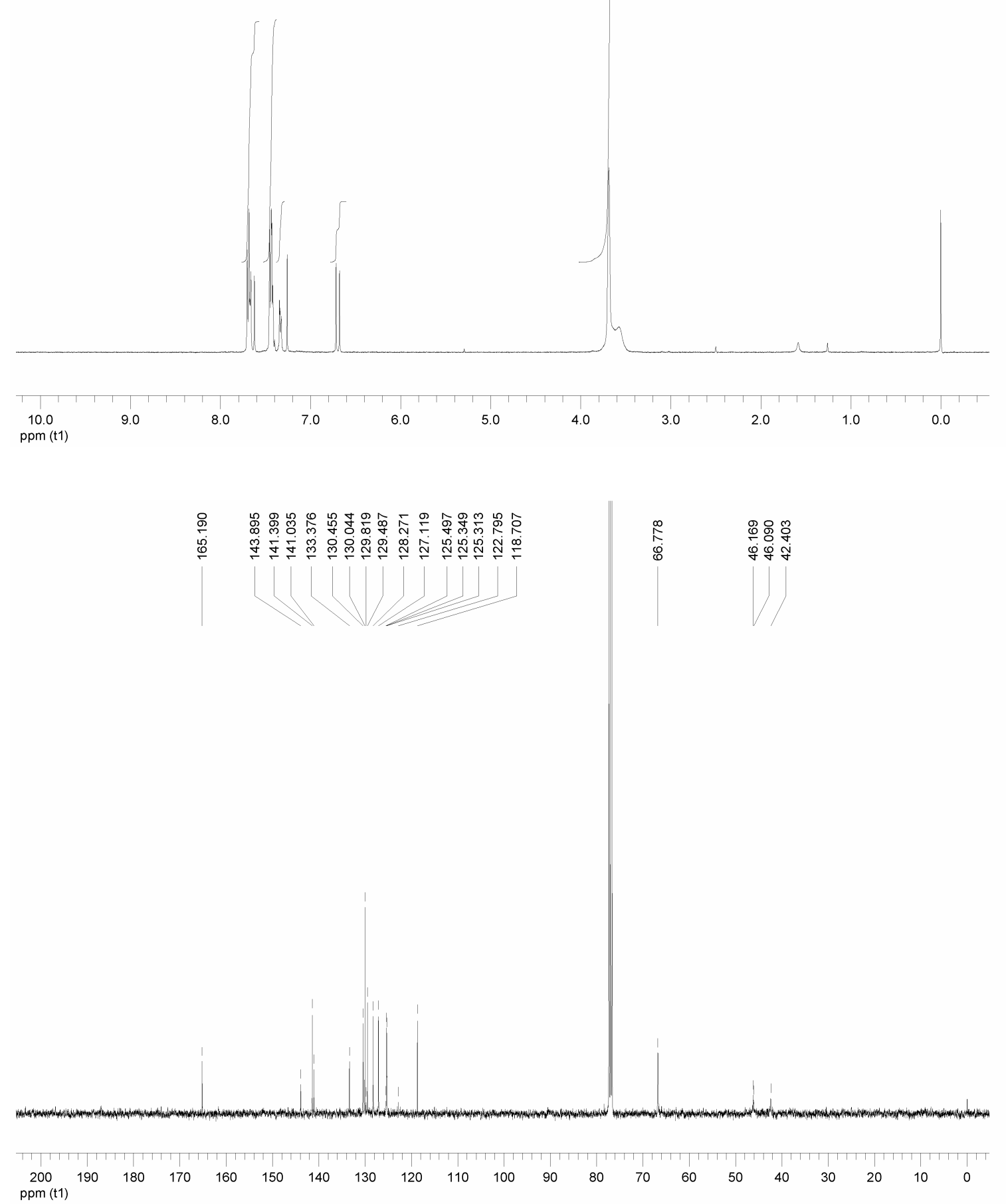


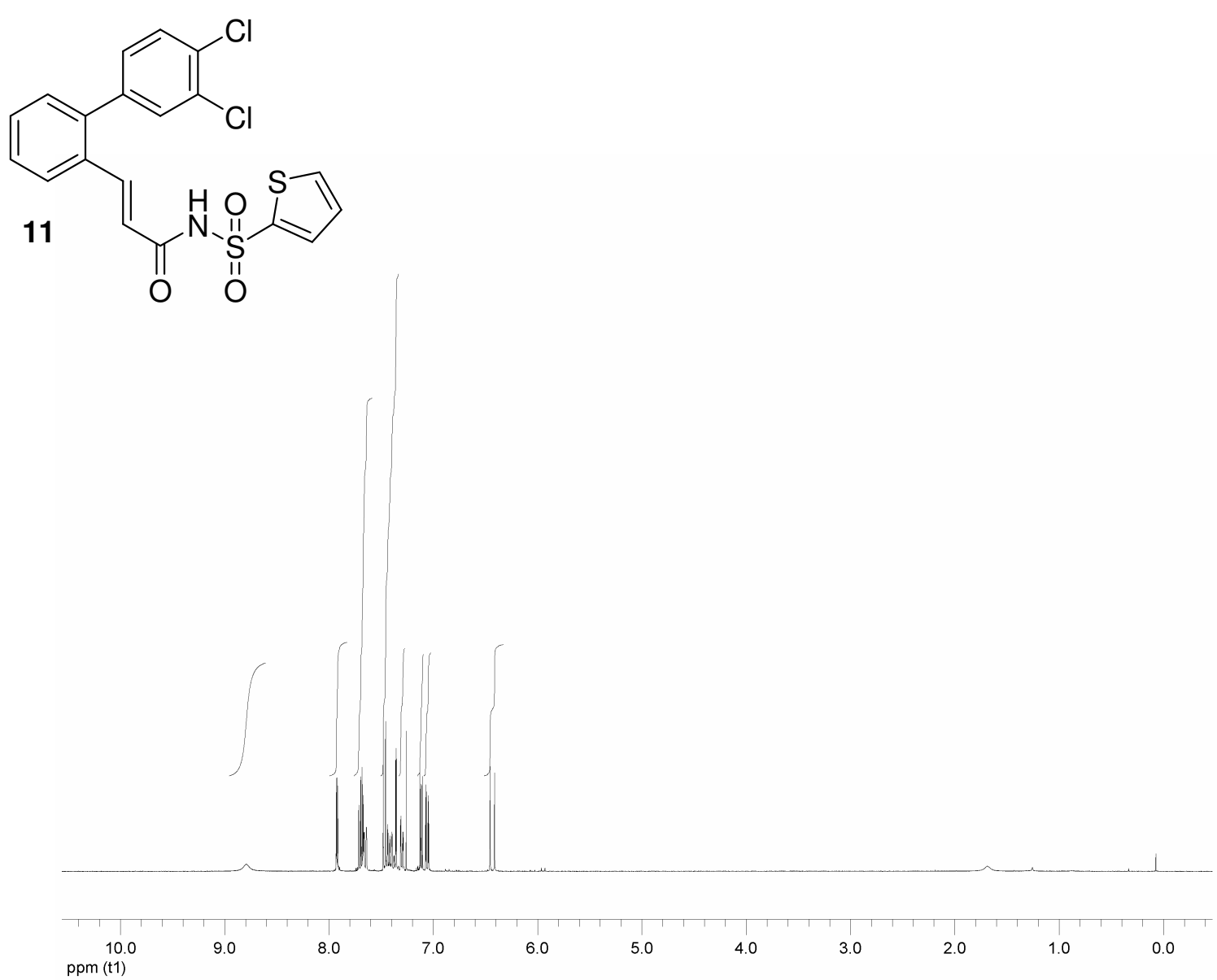

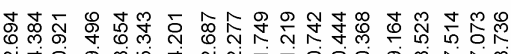

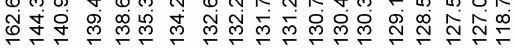

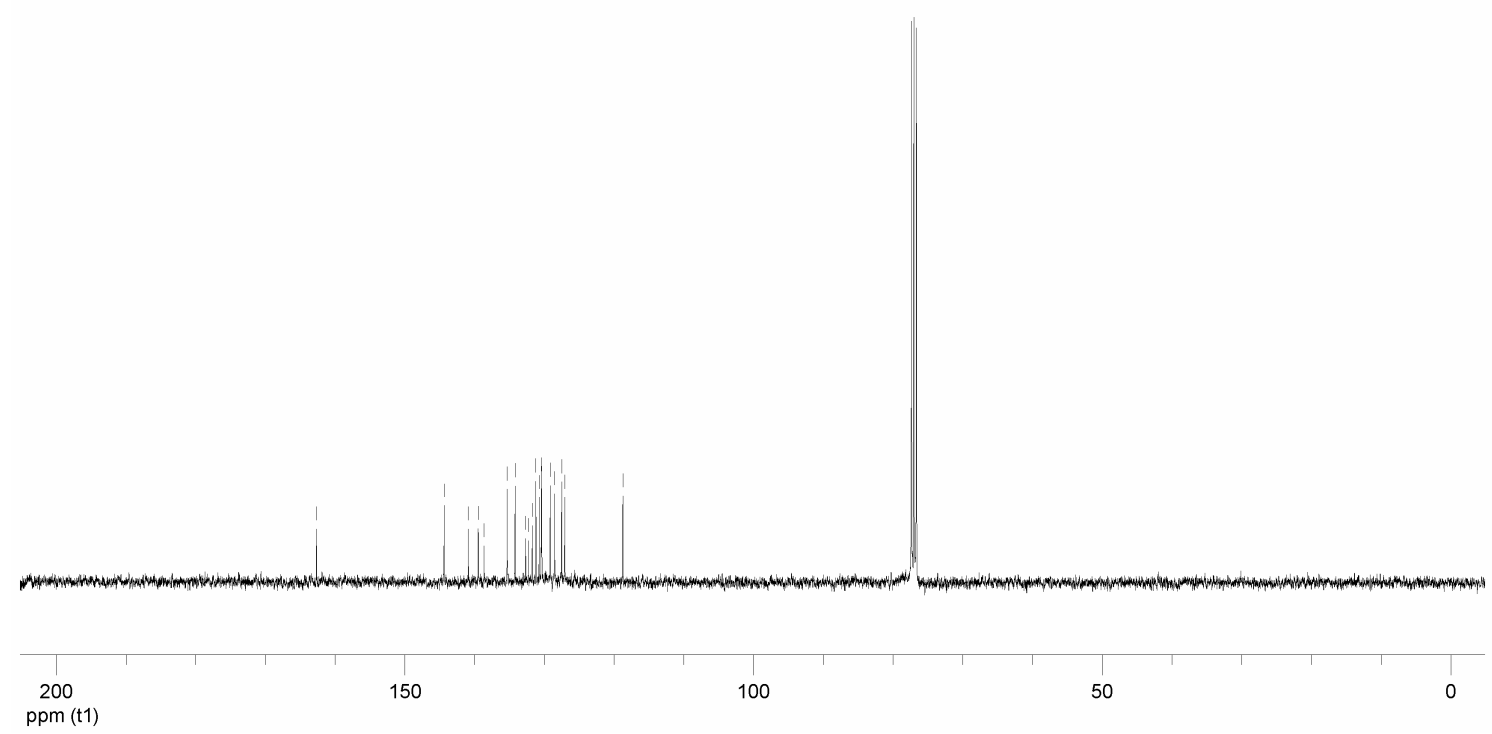


<smiles>CCCCOC(=O)/C=C/c1c(OC)cccc1-c1ccc(C(F)(F)F)cc1</smiles>
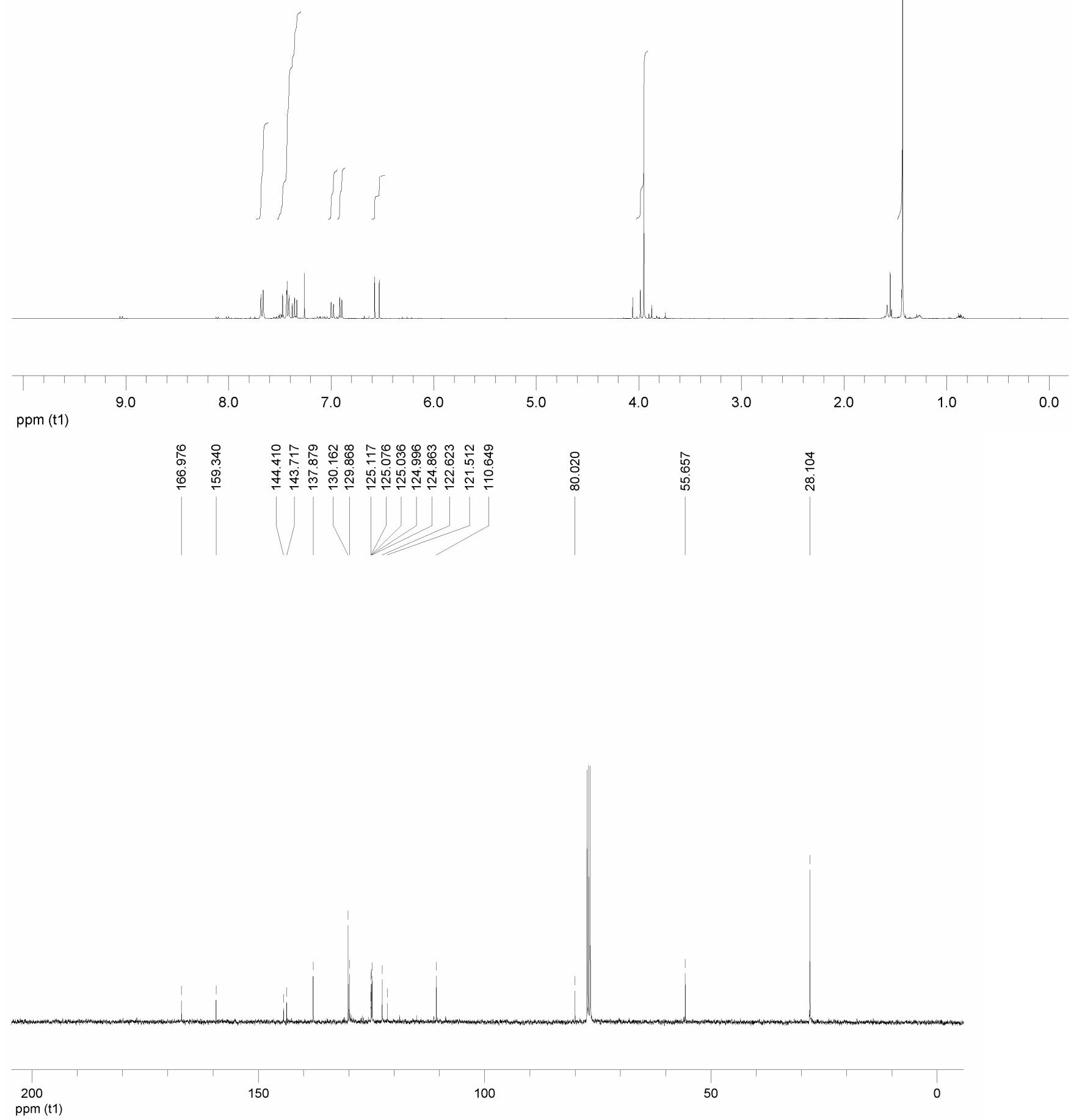

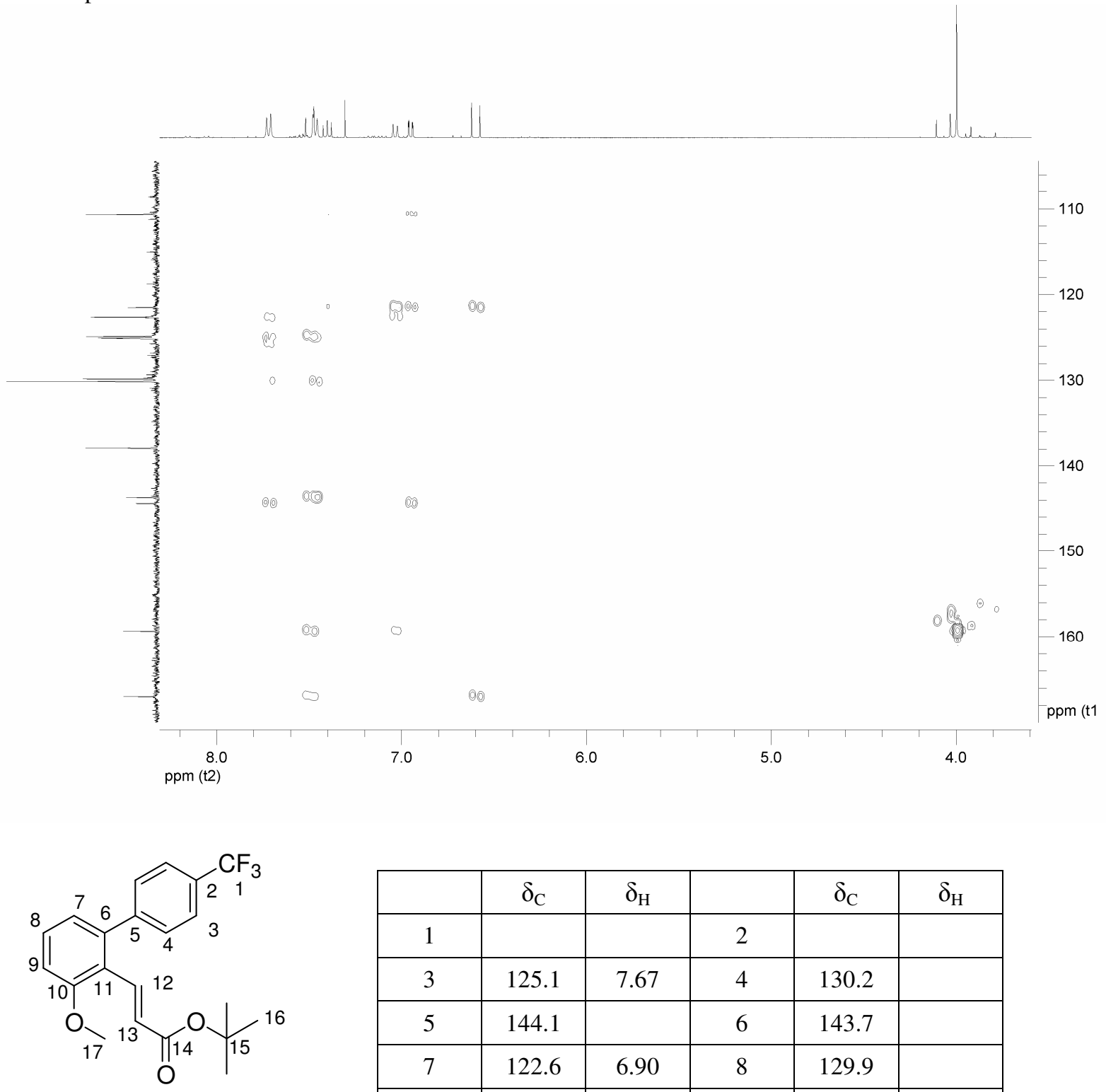

\begin{tabular}{|c|c|c|c|c|c|}
\hline & $\delta_{\mathrm{C}}$ & $\delta_{\mathrm{H}}$ & & $\delta_{\mathrm{C}}$ & $\delta_{\mathrm{H}}$ \\
\hline 1 & & & 2 & & \\
\hline 3 & 125.1 & 7.67 & 4 & 130.2 & \\
\hline 5 & 144.1 & & 6 & 143.7 & \\
\hline 7 & 122.6 & 6.90 & 8 & 129.9 & \\
\hline 9 & 110.6 & 6.99 & 10 & 159.3 & \\
\hline 11 & 121.5 & & 12 & 137.9 & 7.47 \\
\hline 13 & 124.9 & 6.55 & 14 & 167.0 & \\
\hline 15 & 80.0 & & 16 & 28.1 & 1.43 \\
\hline 17 & 55.7 & 3.95 & & & \\
\hline
\end{tabular}

Diagnostic three bond couplings: $\quad \mathrm{H} 9$ to $\mathrm{C} 11$ and $\mathrm{C} 7$

$\mathrm{H} 7$ to $\mathrm{C} 9, \mathrm{C} 11, \mathrm{C} 5$

$\mathrm{H} 13$ to $\mathrm{C} 11$

$\mathrm{H} 3$ to $\mathrm{C} 5$

$\mathrm{H} 12$ to $\mathrm{C} 10$

Alternative regioisomer (14a connectivity in Scheme 4) would show three bond couplings from $\mathrm{H} 12$ to a $\mathrm{CH}$ carbon and absence of 3-bond coupling from $\mathrm{H} 7$ to $\mathrm{C} 5$. 
<smiles>CCOC(=O)/C=C/c1c(-c2ccc(C(F)(F)F)cc2)ccc(OC)c1OC</smiles>

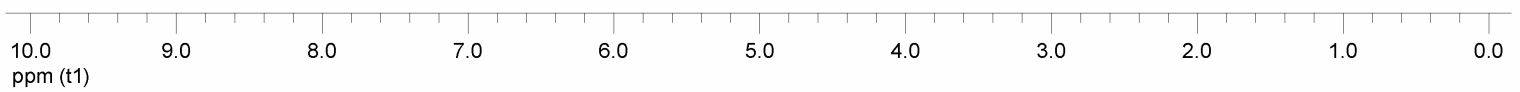

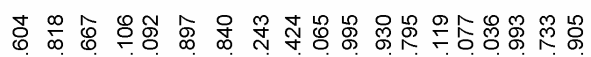

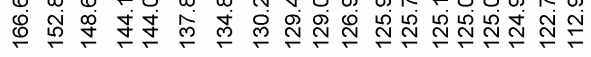
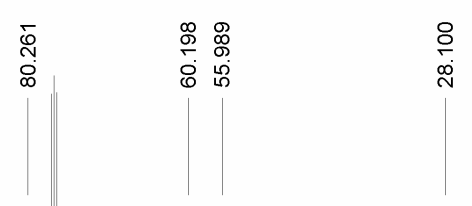

ppm (t1)

100 

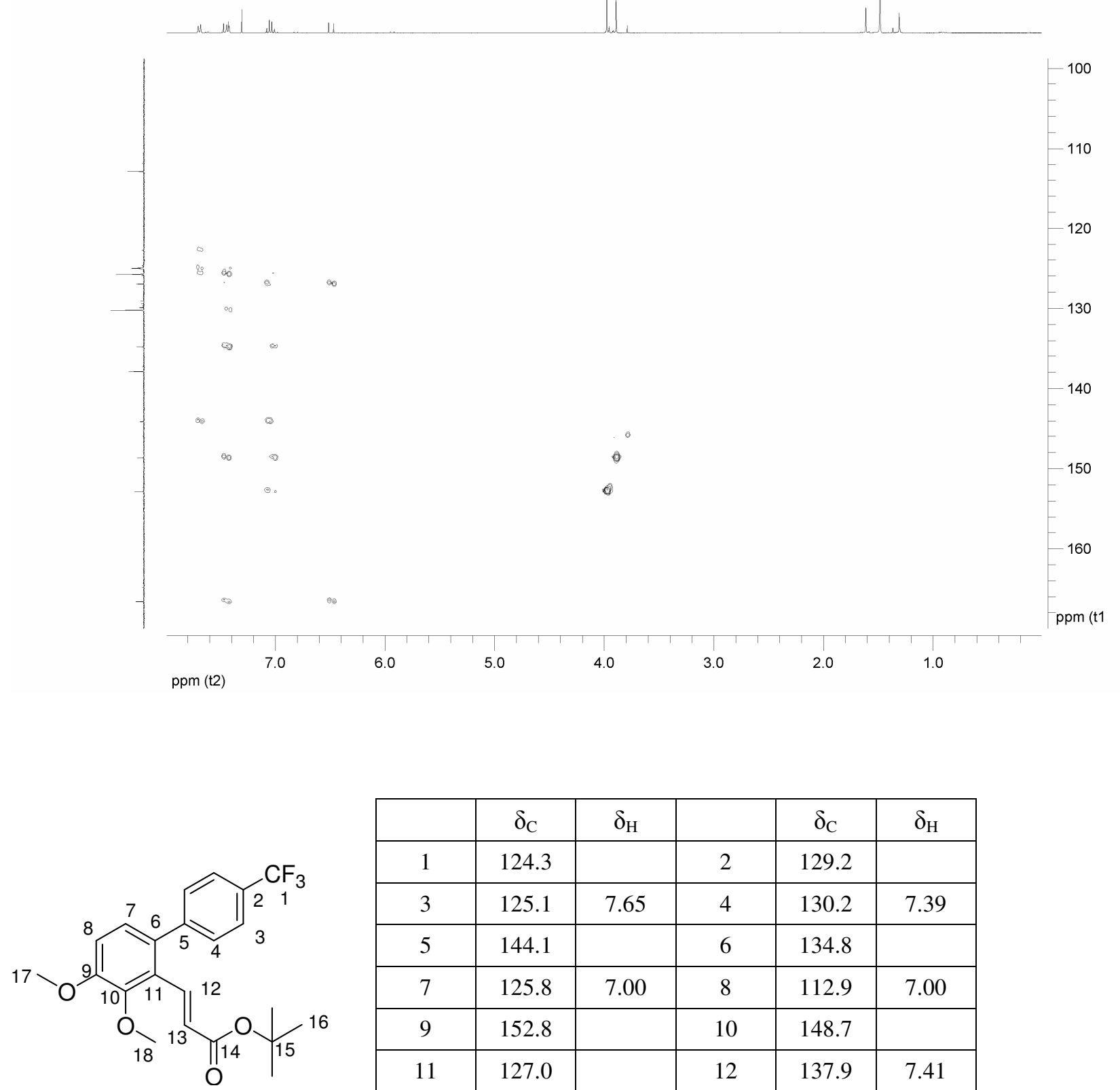

\begin{tabular}{|c|c|c|c|c|c|}
\hline & $\delta_{\mathrm{C}}$ & $\delta_{\mathrm{H}}$ & & $\delta_{\mathrm{C}}$ & $\delta_{\mathrm{H}}$ \\
\hline 1 & 124.3 & & 2 & 129.2 & \\
\hline 3 & 125.1 & 7.65 & 4 & 130.2 & 7.39 \\
\hline 5 & 144.1 & & 6 & 134.8 & \\
\hline 7 & 125.8 & 7.00 & 8 & 112.9 & 7.00 \\
\hline 9 & 152.8 & & 10 & 148.7 & \\
\hline 11 & 127.0 & & 12 & 137.9 & 7.41 \\
\hline 13 & 125.8 & 6.45 & 14 & 166.6 & \\
\hline 15 & 80.3 & & 16 & 28.1 & 1.44 \\
\hline 17 & 56.0 & 3.94 & 18 & 60.2 & 3.85 \\
\hline
\end{tabular}

Diagnostic three bond couplings: $\quad \mathrm{H} 12$ to $\mathrm{C} 10$ and C6

$\mathrm{H} 7$ to $\mathrm{C} 5$

$\mathrm{H} 8$ to $\mathrm{H} 10$ and $\mathrm{H} 6$

Alternative regioisomer (14a connectivity in Scheme 4) would show three bond couplings from H12 to a CH carbon and vice verca, from an aryl $\mathrm{H}$ to $\mathrm{C} 12$. 
Part E. X-ray structure of acid derivative of 20<smiles>COc1ccc(-c2ccc(C(F)(F)F)cc2)c(/C=C/C(=O)O)c1OC</smiles>

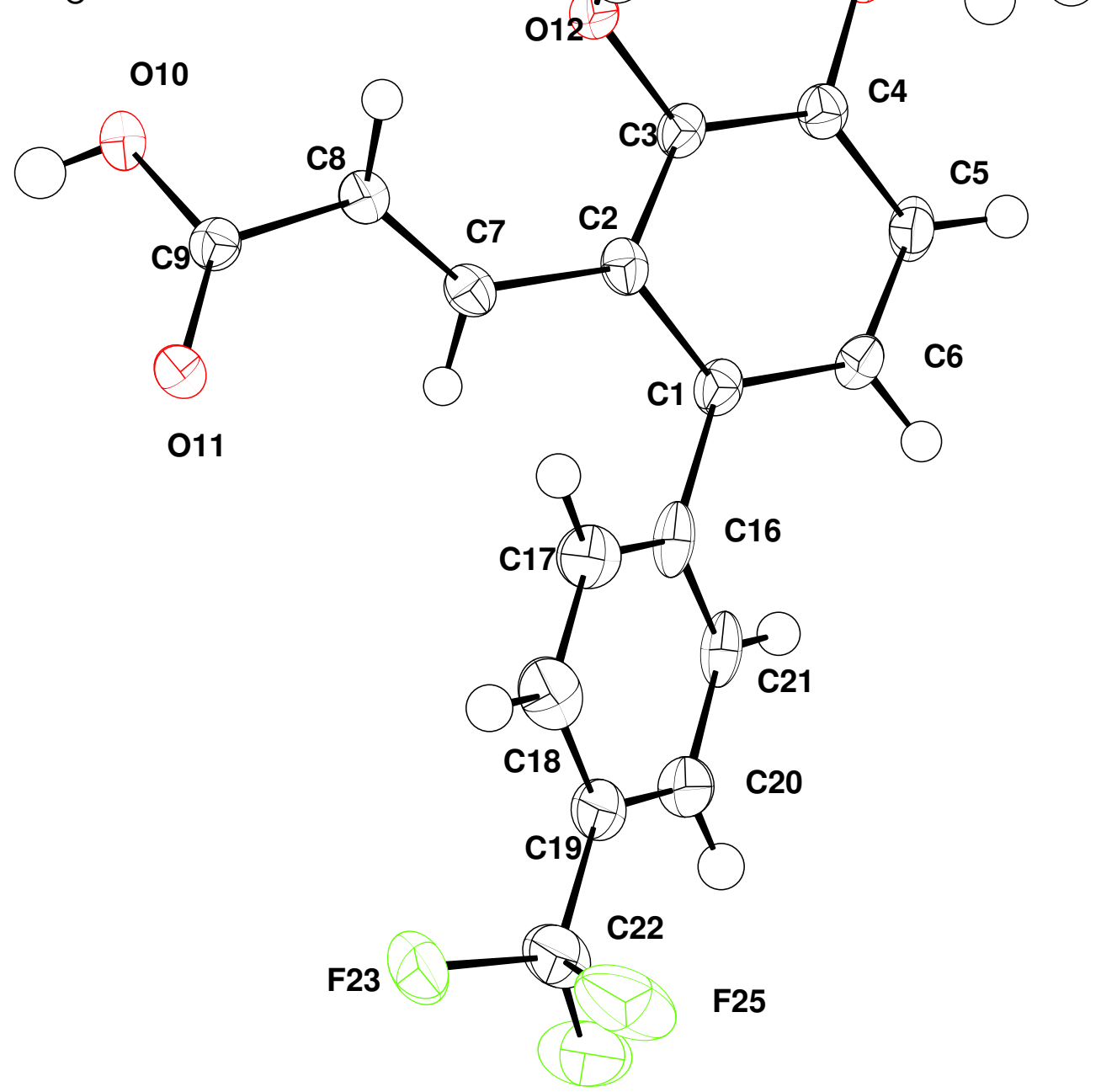

F24 